

\title{
Homotopy exact sequences and orbifolds
}

\author{
Kentaro Mitsui
}

\begin{abstract}
We generalize the homotopy exact sequences of étale fundamental groups for proper separable fibrations to the case where fibrations are not necessarily proper and separable. To treat the case where fibrations admit nonreduced geometric fibers, we introduce orbifolds within the framework of schemes and study their fundamental groups. As an application, we give a criterion for simpleconnectedness of elliptic surfaces over an algebraically closed field by classifying simply connected orbifold curves.
\end{abstract}

1. Introduction 1089

2. Notation and conventions 1094

3. Orbifolds 1095

4. Homotopy exact sequences 1100

5. Orbifold trivializations of orbifold curves 1110

6. Fundamental groups of elliptic fibrations 1118

Appendix A. Triangle groups and projective special linear groups 1130

Appendix B. Comparison between orbifolds and stacks 1133

Acknowledgments 1134

References 1134

\section{Introduction}

In algebraic geometry, the determination of fundamental groups of algebraic varieties is a classical problem. However, the problem is difficult, especially in the positivecharacteristic case, where few results are known except for the one-dimensional case. In this paper, we develop a method to compute étale fundamental groups of fibered regular schemes, and apply the method to study elliptic surfaces, which provides insight into the computation of étale fundamental groups of fibered varieties.

We denote the étale fundamental group of a pointed connected locally Noetherian scheme $(X, \bar{x})$ by $\pi_{1}(X, \bar{x})$. In the introduction, we omit the geometric point $\bar{x}$ for simplicity. Let $f: X \rightarrow S$ be a proper separable morphism between connected

MSC2010: primary 14F35; secondary 14D06, 14J27, 14H30.

Keywords: orbifolds, elliptic surfaces, coverings of curves, étale fundamental groups, homotopy exact sequences, simply connected. 
locally Noetherian schemes with $\mathscr{O}_{S}=f_{*} \mathcal{O}_{X}$. The fibration $f: X \rightarrow S$ may be characterized by the following conditions:

(1) $S$ is a connected locally Noetherian scheme.

(2) $f: X \rightarrow S$ is a faithfully flat proper morphism.

(3) The homomorphism $\mathrm{O}_{S} \rightarrow f_{*} \mathrm{O}_{X}$ associated to $f$ is an isomorphism.

(4) Any geometric fiber of $f$ is reduced.

Choose a geometric fiber $i: X_{0} \rightarrow X$ of $f$. In this case, Grothendieck showed in [SGA 1 1971, X.1] that the morphisms $i$ and $f$ induce a homotopy exact sequence

$$
\pi_{1}\left(X_{0}\right) \stackrel{i_{*}}{\longrightarrow} \pi_{1}(X) \stackrel{f_{*}}{\longrightarrow} \pi_{1}(S) \longrightarrow 1 \text {. }
$$

However, Condition (4) is too strong to compute fundamental groups of fibered varieties, e.g., elliptic surfaces, which may admit nonreduced geometric fibers.

Introducing orbifolds and their fundamental groups, we generalize the above homotopy exact sequence to the case where fibrations admit nonreduced geometric fibers. Instead of considering all general connected locally Noetherian schemes, we restrict ourselves to regular integral schemes. We consider a fibration $f: X \rightarrow S$ satisfying the following conditions (see Definition 4.17 for slightly weaker conditions):

(1') $X$ and $S$ are regular integral schemes.

(2') $f: X \rightarrow S$ is a faithfully flat morphism of finite type.

$\left(3^{\prime}\right) \mathrm{O}_{S}$ is integrally closed in $f_{*} \mathrm{O}_{X}$.

$\left(4^{\prime}\right)$ The geometric generic fiber of $f$ is reduced.

For example, all elliptic fibrations over curves satisfy these conditions (Section 6A). In order to give a similar exact sequence with the same homomorphism $i_{*}$ in the general case (Section $4 \mathrm{C}$ ), we have to replace $\pi_{1}(S)$ by its extension. To this end, we introduce orbifolds within the framework of schemes and study their fundamental groups (Section 3).

An orbifold $(S, B)$ is defined as a locally Noetherian normal scheme $S$ with data of ramifications $B$ (Definition 3.6). Any orbifold curve over an algebraically closed field may be regarded as a DM stack (Theorem B.1). However, in the higher dimensional case, our orbifolds are different from DM stacks and more suitable than DM stacks for our studies (Remark B.2). We denote the fundamental group of a pointed orbifold $(S, B, \bar{s})$ by $\pi_{1}(S, B, \bar{s})$ (Definition 3.22). The local invariants associated to the nonreduced geometric fibers of $f$ (Section 4B) endow $S$ with an orbifold structure. Our main result is the following (Section 4C): 
Theorem 1.1. Let $(X, S, f)$ be a triple satisfying Condition $\left(C^{*}\right)$ (Definition 4.17). Take the orbifold $(S, B)$ associated to $f$ (Definition 4.23). Choose a connected reduced geometric fiber $i: X_{0} \rightarrow X$ of $f$ over a regular point (e.g., the geometric generic fiber of $f$ ). Take a geometric point $\bar{x}_{0}$ on $X_{0}$. Put $\bar{x}:=i\left(\bar{x}_{0}\right)$ and $\bar{s}:=f(\bar{x})$. The morphisms $i$ and $f$ induce canonical homomorphisms $i_{*}: \pi_{1}\left(X_{0}, \bar{x}_{0}\right) \rightarrow$ $\pi_{1}(X, \bar{x})$ and $f_{*}^{\text {orb }}: \pi_{1}(X, \bar{x}) \rightarrow \pi_{1}(S, B, \bar{s})$, respectively (Definition 4.25). Then the sequence

$$
\pi_{1}\left(X_{0}, \bar{x}_{0}\right) \stackrel{i_{*}}{\longrightarrow} \pi_{1}(X, \bar{x}) \stackrel{f_{*}^{\text {orb }}}{\longrightarrow} \pi_{1}(S, B, \bar{s}) \longrightarrow 1
$$

is exact.

Next, we apply the above theorem to the case where $f: X \rightarrow S$ is the structure morphism of an elliptic surface over an algebraically closed field (Section 6). We determine the data of ramifications $B$ of the orbifold $(S, B)$ induced by $f$ (Section 6B), and determine which orbifold is induced by an elliptic surface (Section 6C). As a result, we obtain a criterion for simple-connectedness of elliptic surfaces (Section 6D):

Theorem 1.2. Let $k$ be an algebraically closed field of characteristic $p \geq 0$. Let $C$ be a connected proper smooth $k$-curve. Let $(X, C, f)$ be a relatively minimal elliptic fibration (Definition 6.1). For each closed point s on $C$, we set

$$
m_{s}:= \begin{cases}m & \text { if } f^{-1}(s) \text { is of type }{ }_{m} \mathrm{I}_{n}(n \geq 0) \text { (the Kodaira symbol) } \\ 1 & \text { otherwise. }\end{cases}
$$

By $n_{s}$ we denote the maximum integer satisfying $p \nmid n_{s}$ and $n_{s} \mid m_{s}$ (if $p=0$, then $\left.n_{s}=m_{s}\right)$. Then $X$ is simply connected if and only if all of the following conditions are satisfied:

(1) $\chi\left(\mathbb{O}_{X}\right)>0$.

(2) $C \cong \mathbb{P}_{k}^{1}$.

(3) $\#\left\{s \in C(k) \mid n_{s}>1\right\} \leq 2$.

(4) $\operatorname{gcd}\left(n_{s}, n_{t}\right)=1$ for $s \neq t$.

(5) If $p>0$ and $f^{-1}(s)$ is of type ${ }_{m} \mathrm{I}_{n}(n>0)$, then $p \nmid m$.

(6) If $p>0$ and $\left(f^{-1}(s)\right)_{\text {red }}$ is isomorphic to an ordinary elliptic curve, then the $\mathrm{O}_{C, s}$-module $\left(R^{1} f_{*} \mathrm{O}_{X}\right)_{s}$ is torsion-free.

Furthermore, each of Conditions (1)-(6) is necessary.

We make a remark on Conditions (5) and (6) in the above theorem, which appear only in the positive-characteristic case. Under certain technical assumptions, Katsura and Ueno [1985, §§6-7] observed that an elliptic surface admits a nontrivial étale covering if one of Conditions (5) and (6) is not satisfied. Localizing elliptic 
surfaces with respect to the base curve and using Galois cohomology groups, we study this phenomenon in a systematic way (Section 6B).

We may give plenty of examples of elliptic fibrations with multiple fibers by means of the algebraic analog [Lang 1986; Cossec and Dolgachev 1989, V, §4] (Section 6C) and the rigid analytic analog [Mitsui 2013] of Kodaira's logarithmic transformation [1964, §4] for complex analytic elliptic fibrations. As for topological fundamental groups in the complex analytic case, Moishezon [1977, Theorem 11, II, §2, p. 191] gave a similar criterion by means of deformations of elliptic fibrations in the category of differentiable manifolds. Although the determination of topological fundamental groups is an old problem, no references can be found for étale fundamental groups. Our proof is purely algebraic and applies in any characteristic.

Let us briefly review the studies on the fundamental group of an elliptic surface $f: X \rightarrow C$ in the complex analytic case. Take a smooth fiber $i: X_{0} \rightarrow X$ of $f$. The morphism $i$ induces a homomorphism $i_{*}: \pi_{1}\left(X_{0}\right) \rightarrow \pi_{1}(X)$. In order to study $\pi_{1}(X)$, Iitaka [1971, §4] determined Coker $i_{*}$. He reduced the problem to the case where $X$ does not admit any multiple fiber by using Kodaira's logarithmic transformation and van Kampen's theorem. In a similar way, Xiao [1991] studied the case of more general compact complex analytic fibered surfaces. In another point of view, the group Coker $i_{*}$ is relatively easy to deal with because it may be interpreted as the fundamental group of the orbifold curve induced by the elliptic fibration $f$ [Ue 1986, $§ 1$; Friedman and Morgan 1994, 1.3.6]. If $\chi\left(0_{X}\right)=0$, then the map $f$ between the underlying topological spaces may be regarded as a higher dimensional analog of a Seifert fibration [Seifert 1933; Thornton 1967]. Thurston [1980, §13.4] studied circle bundles over two-dimensional orbifolds in the context of the geometry of three-manifolds, which clarified the structure of Seifert fibrations: a Seifert fibration may be regarded as a circle bundle over a two-dimensional orbifold. After these studies, Ue [1986, $\S 1]$ showed that $\pi_{1}(X)$ is isomorphic to the fundamental group of the orbifold curve induced by $f$ whenever $\chi\left(O_{X}\right)>0$. Using the orbifold curve, Friedman and Morgan [1994, 2.2.1 and 2.7.2] discussed the general case in a systematic way. In the present paper, we develop this idea of using orbifolds within the framework of schemes, and give homotopy exact sequences as explained above.

As for $\operatorname{Im} i_{*}$, no difference appears between the characteristic-zero case and the positive-characteristic case (Theorem 6.23). However, some differences appear between the algebraic case of characteristic zero and the complex analytic case (Remark 6.24). As for Coker $i_{*}$, no difference appears between the algebraic case of characteristic zero and the complex analytic case. As mentioned above, the group Coker $i_{*}$ is isomorphic to the fundamental group of the orbifold curve induced by $f$. Thus, it follows from the fact that any compact complex analytic curve is algebraic. 
However, several differences appear between the characteristic-zero case and the positive-characteristic case, as explained below.

In the algebraic case of characteristic zero and in the complex analytic case, the local structure of the orbifold curve is determined by the multiplicities of the multiple fibers. On the other hand, in the positive-characteristic case, the local structure is more complicated. This is because the completion of the local ring of the base curve at any point admits lots of finite coverings even if we fix the degree of the covering. For example, the completion admits infinitely many Artin-Schreier coverings. In particular, the resolution of multiple fibers in the positive characteristic case is much more difficult [Katsura and Ueno 1985, §§6-7; 1986, §2; Liu et al. $2004, \S 8.6]$ than that in the algebraic case of characteristic zero and in the complex analytic case [Kodaira 1963, §6]. In order to determine the local structure of the orbifold, we develop the above resolution of multiple fibers and study the minimal regular models of torsors of elliptic curves (Section 6B). In conclusion, multiple fibers of additive type [Katsura and Ueno 1986] do not affect the local structure of the orbifold, and the local uniformizations of the orbifold are given by certain finite cyclic coverings (Proposition 6.11).

In order to show Theorem 1.2, we classify simply connected orbifold curves that are locally uniformized by finite cyclic coverings (Theorem 1.3(1)). More precisely, we prove a generalized Fenchel conjecture (Theorem 1.3(2)). The original conjecture states that any finitely generated Fuchsian group admits a torsion-free subgroup of finite index, which was proved by a purely group-theoretic approach in [Fox 1952] and [Chau 1983]. The conjecture is equivalent to the following: any compact complex analytic orbifold curve minus finitely many points may be trivialized by a finite branched covering except for some trivial cases. In other words, except for some trivial cases, there exists a finite branched covering of a given compact complex analytic curve minus finitely many points with prescribed ramifications. Using the geometry of orbifold curves, we generalize this result in any characteristic (Section 5):

Theorem 1.3. Let $(C, B)$ be a connected cyclic orbifold $k$-curve (Definition 5.1). Take the tame part $\left(C, B^{t}\right)$ and the wild part $\left(C, B^{w}\right)$ of $(C, B)$ (Definition 3.8). Put $M:=$ \#Supp $B$ and $N:=$ \# Supp $B^{t}$ (Definition 3.6). For each $s \in \operatorname{Supp} B^{t}$, we put $n_{s}:=\left[B_{s}^{t}: K_{S}\right]$. Then:

(1) The orbifold $(C, B)$ is simply connected (Definition 3.19) if and only if one of the following conditions is satisfied:

(a) $C \cong \mathbb{A}_{k}^{1}, M=0$, and $p=0$.

(b) $C \cong \mathbb{P}_{k}^{1}, B^{t}=B, M \leq 2$, and $\operatorname{gcd}\left(n_{s}, n_{t}\right)=1$ for $s \neq t$.

(2) There exists an orbifold trivialization of $(C, B)$ (Definition 3.10) if and only if neither of the following conditions are satisfied: 
(a) $C \cong \mathbb{P}_{k}^{1}, M=N=1$, and $e\left(C, B^{w}\right)>0$ (Definition 5.14).

(b) $C \cong \mathbb{P}_{k}^{1}, B^{t}=B, M=2$, and $n_{s} \neq n_{t}$ where $\operatorname{Supp} B=\{s, t\}$.

Let us explain our proof. The problem may be reduced to showing the existence or nonexistence of the following four types of coverings:

(1) a covering of the projective line with at most two tame branch points;

(2) a covering of the projective line with three tame branch points;

(3) a covering of a curve with one tame branch point;

(4) a covering of a curve with one wild branch point.

Case (1) is easy. In the other cases, difficulties arise when we construct a covering of a curve with prescribed ramifications. In Case (2), we use the techniques of degeneration of a covering of a curve over a mixed characteristic ring [Raynaud 1994, §6]. In Cases (3) and (4), we produce rational functions on étale coverings with prescribed zeros and poles in order to apply Kummer theory and Artin-SchreierWitt theory.

Finally, the classification of simply connected orbifolds and the above studies on the homotopy exact sequences give the desired criterion for simple-connectedness of elliptic surfaces.

\section{Notation and conventions}

We denote the cardinality of a set $A$ by \# $A$ and the degree of a finite field extension $L / K$ by $[L: K]$. We denote the field of fractions of an integral domain $R$ by Frac $R$ and the strict Henselization of a local ring $R$ by $R^{\text {sh }}$. For a ring $R$, an $R$-curve is a faithfully flat separated $R$-scheme of finite type and of pure relative dimension one. We denote the geometric genus of a proper curve $C$ over a field by $g(C)$. The multiplicity of a nonzero Weil divisor $D$ on a locally Noetherian normal scheme $X$ is the maximum positive integer $m$ such that there exists a Weil divisor $D^{\prime}$ on $X$ satisfying $D=m D^{\prime}$. A scheme $Y$ over a scheme $X$ is called an étale covering space of $X$ if the structure morphism $Y \rightarrow X$ is finite, étale, and surjective. A scheme $X$ is said to be simply connected if $X$ is connected and does not admit any nontrivial connected étale covering space of $X$.

Let $X$ be a connected locally Noetherian scheme. Take a geometric point $\bar{x}:$ Spec $\Omega \rightarrow X$ on $X$, where $\Omega$ is a separably closed field. The pair $(X, \bar{x})$ is called a pointed connected locally Noetherian scheme. We denote the étale fundamental group of $(X, \bar{x})$ by $\pi_{1}(X, \bar{x})$. We sometimes omit $\bar{x}$ and denote $\pi_{1}(X, \bar{x})$ by $\pi_{1}(X)$ for simplicity. 


\section{Orbifolds}

Definition 3.1. A morphism $f$ between schemes is said to be separable if $f$ is flat and the fiber of $f$ over any point is geometrically reduced [SGA 1 1971, X.1.1]. A morphism $f: X \rightarrow Y$ between schemes is said to be generically separable if $f$ maps any point $x$ of codimension zero to a point $y$ of codimension zero and induces a separable morphism Spec $\mathrm{O}_{X, x} \rightarrow \operatorname{Spec} \mathrm{O}_{Y, y}$. A morphism $f: X \rightarrow Y$ between schemes is called a quasiseparable-covering (qsc) morphism if $f$ is a locally quasifinite generically separable morphism. We say that a morphism $f$ between locally Noetherian schemes preserves codimensions if $f$ maps any point to a point of the same codimension.

Remark 3.2. A separable-covering morphism between integral schemes is conventionally defined as a finite generically separable morphism. The notion of a qsc morphism is a generalization of this notion.

We frequently use the following:

Lemma 3.3. (1) Any locally Noetherian normal scheme is the disjoint union of locally Noetherian integral schemes [Matsumura 1989, Exercise 9.11].

(2) The normalization of any locally Noetherian normal integral scheme $X$ in any finite separable field extension of the function field of $X$ is finite over $X$ [Matsumura 1989, §33, Lemma 1].

(3) Any separated qsc morphism $X \rightarrow Y$ between connected locally Noetherian normal schemes decomposes into an open immersion $X \rightarrow Z$ and a finite qsc morphism $Z \rightarrow Y$ where $Z$ is a connected locally Noetherian normal scheme $\left[\mathrm{EGA} \mathrm{IV}_{3}\right.$ 1966, 8.12.11].

Lemma 3.4 [Matsumura 1989, 9.4 and 15.1]. Let $\phi: A \rightarrow B$ be a homomorphism between Noetherian rings, and $Q$ a prime ideal of $B$. Put $P:=\phi^{-1}(Q)$. Then:

(1) ht $Q \leq$ ht $P+\operatorname{dim} B_{Q} / P B_{Q}$.

(2) If $\phi$ is flat, then the equality in (1) holds.

(3) Any qsc morphism between locally Noetherian normal schemes preserves codimensions.

Lemma 3.5 (Zariski-Nagata purity [SGA 1 1971, X.3.1]). Let $f: X \rightarrow Y$ be a qsc morphism between locally Noetherian schemes. Assume that $X$ is normal and $Y$ is regular. Then any irreducible component of the non-étale locus of $f$ is of codimension one.

Definition 3.6. Let $S$ be a locally Noetherian normal scheme. By $P(S)$ we denote the set of all points on $S$ of codimension one. For each $s \in P(S)$ put $K_{S}:=$ Frac $0_{S, s}^{\text {sh }}$. Take a separable closure $\overline{K_{s}}$ of $K_{s}$. Let $B$ be a map that associates $s \in P(S)$ with 
a finite Galois extension $B_{S} / K_{S}$ in $\overline{K_{S}}$ and satisfies the following condition: let Supp $B:=\left\{s \in P(S) \mid B_{s} \neq K_{s}\right\}$; then Supp $B$ is locally finite. The pair $(S, B)$ is called an orbifold. For a locally Noetherian normal scheme $S$, we denote the orbifold obtained by equipping $S$ with the trivial orbifold structure by $(S)$. Let $P$ be a property of schemes (e.g., connected, quasicompact, regular). We say that an orbifold $(S, B)$ is $P$ if $S$ has the property $P$. Let $P$ be a property of finite field extensions (e.g., trivial, tame, wild, cyclic). We say that an orbifold $(S, B)$ is $P$ if $B_{S} / K_{s}$ has the property $P$ for any $s \in P(S)$.

Remark 3.7. In the above definition, we restrict ourselves to the case where the data of ramifications are given only in codimension one for our purpose of application to homotopy exact sequences (see Remark 4.26 for a generalization).

Definition 3.8. Let $(S, B)$ be an orbifold. By $B^{t}$ we denote the map that associates $s \in P(S)$ with the maximal tame field extension $B_{s}^{t} / K_{S}$ in $B_{s}$. Then $\left(S, B^{t}\right)$ is a cyclic orbifold. The orbifold $\left(S, B^{t}\right)$ is called the tame part of $(S, B)$. Assume that $(S, B)$ is cyclic. By $B^{w}$ we denote the map that associates $s \in P(S)$ with the minimum field extension $B_{s}^{w} / K_{s}$ in $B_{s}$ such that the equality $B_{s}^{t} B_{s}^{w}=B_{s}$ holds. Then $\left(S, B^{w}\right)$ is a cyclic orbifold. The orbifold $\left(S, B^{t}\right)$ is called the wild part of $(S, B)$.

Lemma 3.9. Let $u: S^{\prime} \rightarrow S$ be a qsc morphism between locally Noetherian normal schemes. Take $s^{\prime} \in P\left(S^{\prime}\right)$. Put $s:=u\left(s^{\prime}\right)$. Then $s \in P(S)$. Put $K_{s}:=\operatorname{Frac} O_{S, s}^{\text {sh }}$ and $K_{s^{\prime}}^{\prime}:=$ Frac $O_{S^{\prime}, s^{\prime}}^{\text {sh }}$. Then $u$ induces a finite field extension $K_{s^{\prime}}^{\prime} / K_{s}$.

Proof. We may assume that $S$ and $S^{\prime}$ are affine. The first statement follows from Lemma 3.4(3). The last statement follows from Lemma 3.3(3).

Definition 3.10. Let $(S, B)$ and $\left(S^{\prime}, B^{\prime}\right)$ be two orbifolds. We use the notation introduced in Lemma 3.9. Composing the field extensions $K_{s^{\prime}}^{\prime} / K_{s}$ and $B_{s^{\prime}}^{\prime} / K_{s^{\prime}}^{\prime}$, we obtain a field extension $B_{s^{\prime}}^{\prime} / K_{s}$. Assume that there exists a $K_{s^{\prime}}$-algebra homomorphism $\phi_{s^{\prime}}: B_{S} \rightarrow B_{s^{\prime}}^{\prime}$ for all $s^{\prime} \in P\left(S^{\prime}\right)$. Then $u$ is called an orbifold morphism $\left(S^{\prime}, B^{\prime}\right) \rightarrow(S, B)$. If $\phi_{s^{\prime}}$ is an isomorphism for all $s^{\prime} \in P\left(S^{\prime}\right)$, then $u$ is called an orbifold étale morphism $\left(S^{\prime}, B^{\prime}\right) \rightarrow(S, B)$. If $u$ is finite, orbifold étale, and surjective, then $\left(S^{\prime}, B^{\prime}\right)$ is called an orbifold étale covering space of $(S, B)$. If $\left(S^{\prime}, B^{\prime}\right)$ is a trivial orbifold and an orbifold étale covering space of $(S, B)$, then $u$ is called an orbifold trivialization of $(S, B)$.

Remark 3.11. The homomorphisms $\phi_{S^{\prime}}$ are not part of the data of an orbifold morphism. The image of $\phi_{s^{\prime}}$ does not depend on the choice of $\phi_{s^{\prime}}$ since $B_{S} / K_{S}$ is Galois. The composite of any two orbifold (étale) morphisms is an orbifold (étale) morphism.

Definition 3.12. Let $(S, B)$ be an orbifold. We use the notation introduced in Lemma 3.9. Assume that there exists a $K_{s}$-algebra homomorphism $\psi_{s^{\prime}}: K_{s^{\prime}}^{\prime} \rightarrow B_{s}$ 
for all $s^{\prime} \in P\left(S^{\prime}\right)$ (e.g., $u$ is étale). Since $B_{s} / K_{s^{\prime}}^{\prime}$ is Galois, we may define an orbifold $\left(S^{\prime}, B^{\prime}\right)$ by putting $B_{s^{\prime}}^{\prime}:=B_{S}$ for all $s^{\prime} \in P\left(S^{\prime}\right)$. Then $u$ is an orbifold étale morphism $\left(S^{\prime}, B^{\prime}\right) \rightarrow(S, B)$. We say that $u$ induces an orbifold étale morphism $\left(S^{\prime}, B^{\prime}\right) \rightarrow(S, B)$.

Lemma 3.13. Let $f: X \rightarrow S$ and $g: Y \rightarrow S$ be two qsc morphisms between locally Noetherian normal schemes. Take the normalization $Z$ of $X \times_{S} Y$ and the canonical projections $f^{\prime}: Z \rightarrow Y$ and $g^{\prime}: Z \rightarrow X$. Then $f^{\prime}$ and $g^{\prime}$ are qsc morphisms between locally Noetherian normal schemes.

Proof. We may assume that $X, Y$, and $S$ are affine. By (1) and (3) of Lemma 3.3, we have only to show the case where $f$ and $g$ are finite qsc morphisms between integral schemes. In this case, the lemma follows from Lemma 3.3(2).

Lemma 3.14. Take a separable closure $\bar{L}$ of a field $L$. Let $M$ and $N$ be two finite field extensions of $L$ in $\bar{L}$. Put $P:=M \cap N$ and $Q:=M N$. By $\widetilde{Q}$ we denote the Galois closure of $Q / L$. Then the L-algebra $M \otimes_{L} N$ is L-isomorphic to a finite product of $Q$-subfields of $\widetilde{Q}$. If $M / L$ is Galois then $M \otimes_{L} N \cong Q^{[P: L]}$ over $L$, where the right-hand side is the product of $[P: L]$ copies of $Q$. If $M / L$ and $N / L$ are Galois, then $\widetilde{Q}=Q$.

Proof. This follows from the $L$-algebra isomorphism $M \otimes_{L} N \cong P \otimes_{L} Q$.

By $\mathscr{C}$ (resp. $\mathscr{C}_{\text {ét }}$ ) we denote the category consisting of locally Noetherian normal schemes and qsc morphisms (resp. étale morphisms). By $\mathscr{C}_{\text {orb }}$ (resp. $\mathscr{C}_{\text {orb,ét }}$ ) we denote the category consisting of orbifolds and orbifold morphisms (resp. orbifold étale morphisms). We define a faithful functor $F_{\text {orb }}: \mathscr{C} \rightarrow \mathscr{C}_{\text {orb }}$ by $S \mapsto(S)$. In the

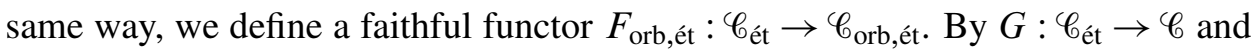
$G_{\text {orb }}: \mathscr{C}_{\text {orb,ét }} \rightarrow \mathscr{C}_{\text {orb }}$ we denote the canonical faithful functors. Then $F_{\text {orb }} \circ G$ is naturally isomorphic to $G_{\text {orb }} \circ F_{\text {orb,ét }}$.

Proposition 3.15. The categories $\mathscr{C}, \mathscr{C}_{\text {ét }}, \mathscr{C}_{\text {orb }}$, and $\mathscr{C}_{\text {orb,ét }}$ admit any finite fiber product. In any case, the (underlying) scheme of any finite fiber product is isomorphic to the normalization of the fiber product of the (underlying) schemes, and any base change of any (orbifold) étale morphism is an (orbifold) étale morphism. The functors $F_{\text {orb }}, F_{\text {orb,ét }}, G$, and $G_{\text {orb }}$ preserve any finite fiber product.

Proof. Let us show the first statement. Lemma 3.13 shows the cases of $\mathscr{C}$ and $\mathscr{C}$ ét. Let $\left(S_{1}, B_{1}\right)$ and $\left(S_{2}, B_{2}\right)$ be two orbifolds over an orbifold $\left(S_{0}, B_{0}\right)$. We define an orbifold $\left(S_{3}, B_{3}\right)$ in the following way. Take the normalization $S_{3}$ of $S_{1} \times S_{0} S_{2}$ and the canonical projection $p_{i}: S_{3} \rightarrow S_{i}$ for $i=0,1$, and 2. Take $s \in P\left(S_{3}\right)$. Put $K_{i}:=\operatorname{Frac} O_{S_{i}, p_{i}(s)}^{\mathrm{sh}}$ and $K_{3}:=$ Frac $O_{S_{3}, s}^{\mathrm{sh}}$. The morphism $p_{i}$ induces a field extension $K_{3} / K_{i}$. Put $L_{i}:=B_{i, p_{i}(s)}$. Take a separable closure $\overline{K_{3}}$ of $K_{3}$. By $L_{i}^{\prime} / K_{3}$ we denote the unique Galois extension in $\overline{K_{3}}$ that is $K_{3}$-isomorphic to the lifting of the Galois extension $L_{i} / K_{i}$ via $K_{3} / K_{i}$. We define $B_{3, s} / K_{3}$ as the Galois 
extension $L_{1}^{\prime} L_{2}^{\prime} / K_{3}$. We apply the same procedure to all $s \in P\left(S_{3}\right)$. Then we obtain an orbifold $\left(S_{3}, B_{3}\right)$ and an orbifold morphism $p_{i}:\left(S_{3}, B_{3}\right) \rightarrow\left(S_{i}, B_{i}\right)$ for $i=0,1$, and 2. By construction, the orbifold $\left(S_{3}, B_{3}\right)$ is the fiber product of $\left(S_{1}, B_{1}\right)$ and $\left(S_{2}, B_{2}\right)$ over $\left(S_{0}, B_{0}\right)$ in $\mathscr{C}_{\text {orb }}$. Thus, the category $\mathscr{C}_{\text {orb }}$ admits any finite fiber product. Assume that $\left(S_{1}, B_{1}\right)$ is orbifold étale over $\left(S_{0}, B_{0}\right)$. By definition, we may regard $L_{0}, L_{1}$, and $L_{2}$ as field extensions of $K_{0}$ satisfying $L_{1}=L_{0} \subset L_{2}$. Since $K_{3} \subset K_{1} \otimes_{K_{0}} K_{2} \subset L_{1} \otimes_{K_{0}} L_{2} \cong L_{2}^{\left[L_{1}: K_{0}\right]}$ (Lemma 3.14), we may regard $K_{3}$ as a $K_{2}$-subfield of $L_{2}$. Then $L_{2} \cong B_{3, s}$ over $K_{2}$. Thus, the morphism $p_{2}:\left(S_{3}, B_{3}\right) \rightarrow\left(S_{2}, B_{2}\right)$ is orbifold étale. In particular, the category $\mathscr{C}_{\text {orb,ét }}$ admits any finite fiber product. The other statements follow from the construction.

Definition 3.16. Let $\left(S, B_{1}\right)$ and $\left(S, B_{2}\right)$ be two orbifolds. For $i=1$ and 2 , the identity on $S$ is an orbifold morphism $\left(S, B_{i}\right) \rightarrow(S)$. We define the composite orbifold $\left(S, B_{1} B_{2}\right)$ of $\left(S, B_{1}\right)$ and $\left(S, B_{2}\right)$ as the fiber product of $\left(S, B_{1}\right)$ and $\left(S, B_{2}\right)$ over $(S)$ in $\mathscr{C}_{\text {orb }}$.

Remark 3.17. By the proof of Proposition 3.15, the field $\left(B_{1} B_{2}\right)_{s}$ is equal to the composite field of $B_{1, s}$ and $B_{2, s}$ for any $s \in P(S)$, where we regard the extensions $B_{1, s}$ and $B_{2, s}$ of $K_{s}$ as subfields of a fixed separable closure $\overline{K_{s}}$.

Proposition 3.18. For $i=1$ and 2 , let $u_{i}:\left(S_{i}\right) \rightarrow\left(S, B_{i}\right)$ be an orbifold trivialization. Take the normalization $S_{3}$ of $S_{1} \times{ }_{S} S_{2}$ and the canonical projection $p_{i}: S_{3} \rightarrow S_{i}$ for $i=1$ and 2 , which is an orbifold morphism $\left(S_{3}\right) \rightarrow\left(S_{i}\right)$. Then the orbifold morphism $\left(S_{3}\right) \rightarrow\left(S, B_{1} B_{2}\right)$ induced by $u_{1} \circ p_{1}$ and $u_{2} \circ p_{2}$ is an orbifold trivialization.

Proof. The proposition follows from Lemma 3.14 and Remark 3.17.

Definition 3.19. An orbifold $(S, B)$ is said to be simply connected if $(S, B)$ is connected and does not admit any nontrivial connected orbifold étale covering space. Let $(S, B)$ be a connected orbifold. Take a geometric point $\bar{s}: \operatorname{Spec} \Omega \rightarrow S$ on $S$, where $\Omega$ is a separably closed field. Assume that the image of $\bar{s}$ is a regular point on $S$ and not contained in the closure of Supp $B$ (e.g., the image is the generic point of $S)$. The triple $(S, B, \bar{S})$ is called a pointed connected orbifold. Let $\mathscr{Y}=(S, B, \bar{s}: \operatorname{Spec} \Omega \rightarrow S)$ and $\mathscr{S}^{\prime}=\left(S^{\prime}, B^{\prime}, \bar{s}^{\prime}: \operatorname{Spec} \Omega^{\prime} \rightarrow S^{\prime}\right)$ be two pointed connected orbifolds. A pointed orbifold (étale) morphism $\mathscr{S}^{\prime} \rightarrow \mathscr{Y}$ is a pair of an orbifold (étale) morphism $u:\left(S^{\prime}, B^{\prime}\right) \rightarrow(S, B)$ and a morphism $\Phi: \operatorname{Spec} \Omega^{\prime} \rightarrow$ Spec $\Omega$ between schemes such that the diagram

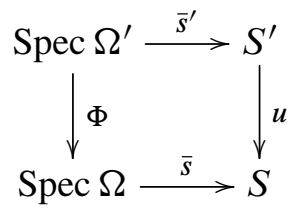

is commutative. 
Remark 3.20. By Zariski-Nagata purity (Lemma 3.5), any orbifold étale morphism to $(S, B)$ is étale over the image of $\bar{s}$.

Let $(S, B, \bar{s})$ be a pointed connected orbifold. By $\mathscr{C}_{(S, B)}$ we denote the category of finite orbifold étale $(S, B)$-orbifolds and orbifold étale $(S, B)$-morphisms. We define a functor $F_{\bar{S}}$ from $\mathscr{C}_{(S, B)}$ to the category of finite sets by sending an object $\left(S^{\prime}, B^{\prime}\right) \rightarrow(S, B)$ to the underlying set of $S^{\prime} \times{ }_{S} \bar{s}$. We refer to [SGA 11971, V.5] for the definition of a Galois category and a fiber functor (a fundamental functor).

Theorem 3.21. The category $\mathscr{C}_{(S, B)}$ is a Galois category with the fiber functor $F_{\bar{s}}$. Proof. We show the theorem in the same way as in the case of the category of finite étale schemes over a pointed connected locally Noetherian scheme (see [SGA 1 1971, V.7]). We have only to verify that the pair $\left(\mathscr{C}_{(S, B)}, F_{\bar{S}}\right)$ satisfies Axioms (G1)-(G6) in [loc. cit., V.4]:

(G1) The orbifold $(S, B)$ is the final object in $\mathscr{C}_{(S, B)}$. The category $\mathscr{C}_{(S, B)}$ admits any finite fiber product (Proposition 3.15).

(G2) The empty set equipped with the trivial orbifold structure is the initial object in $\mathscr{C}_{(S, B)}$. Take an object $u:\left(S^{\prime}, B^{\prime}\right) \rightarrow(S, B)$ in $\mathscr{C}_{(S, B)}$. Assume that a finite group $G$ acts on an orbifold $\left(S^{\prime}, B^{\prime}\right)$ over $(S, B)$ in $\mathscr{C}_{(S, B)}$. By $S^{\prime \prime}$ we denote the spectrum of the ${ }^{0} S$-algebra of $G$-invariant sections of ${ }^{0} S^{\prime}$. Then $S^{\prime \prime}$ is the quotient of $S^{\prime}$ by $G$ in the category of $S$-schemes [loc. cit., V.1.8]. By $v: S^{\prime} \rightarrow S^{\prime \prime}$ we denote the morphism induced by the canonical inclusion homomorphism $0_{S^{\prime \prime}} \rightarrow{ }^{0} S^{\prime}$. The morphism $u$ factors as $u=w \circ v$. Since $u$ is finite, the morphism $v$ is finite. Since $S$ is locally Noetherian and $u$ is finite, the morphism $w$ is finite. Take a point $s^{\prime}$ on $S^{\prime}$ of codimension one. By $I\left(s^{\prime}\right) \subset G$ we denote the inertia group of $s^{\prime}$. We use the notation $B_{s^{\prime}}^{\prime} / K_{s^{\prime}}^{\prime}$ introduced in Definition 3.10. Put $s^{\prime \prime}:=v\left(s^{\prime}\right), B_{s^{\prime \prime}}^{\prime \prime}:=B_{s^{\prime}}^{\prime}$, and $K_{s^{\prime \prime}}^{\prime \prime}:=$ Frac $O_{S^{\prime \prime}, s^{\prime \prime}}^{\mathrm{sh}}$. Then the image under the homomorphism $K_{s^{\prime \prime}}^{\prime \prime} \rightarrow K_{s^{\prime}}^{\prime}$ induced by $v$ is equal to $\left(K_{s^{\prime}}^{\prime}\right)^{I\left(s^{\prime}\right)}$. Furthermore, the extension $B_{s^{\prime \prime}}^{\prime \prime} / K_{s^{\prime \prime}}^{\prime \prime}$ is finite and Galois. By construction, the pair $\left(S^{\prime \prime}, B^{\prime \prime}\right)$ is an orbifold that is a quotient of $\left(S^{\prime}, B^{\prime}\right)$ by $G$ in $\mathscr{C}_{(S, B)}$.

(G3) Lemma 3.3(1) implies that any morphism $u:\left(S^{\prime}, B^{\prime}\right) \rightarrow(S, B)$ in $\mathscr{C}_{(S, B)}$ factors as $w \circ v:\left(S^{\prime}, B^{\prime}\right) \rightarrow\left(S^{\prime \prime}, B^{\prime \prime}\right) \rightarrow(S, B)$ where $v$ is a strict epimorphism, $w$ is a monomorphism, and $\left(S^{\prime \prime}, B^{\prime \prime}\right)$ is a direct summand of $(S, B)$.

(G4) By definition, the functor $F_{\bar{s}}$ is left-exact.

(G5) By definition, the functor $F_{\bar{s}}$ preserves any finite direct sum and preserves the quotient by any action of any finite group. Take a strict epimorphism $u$ in $\mathscr{C}_{(S, B)}$. We have to show that $F_{\bar{S}}(u)$ is surjective. By $s$ we denote the image of $\bar{s}$ on $S$. By base change, we may replace $S$ by Spec $0_{S, s}$. Then $u$ is étale (Remark 3.20). Thus, the surjectivity follows from [loc. cit., V.3.5]. 
(G6) Take a morphism $u$ in $\mathscr{C}_{(S, B)}$. Assume that $F_{\bar{S}}(u)$ is an isomorphism. We have to show that $u$ is an isomorphism. By $s$ we denote the image of $\bar{s}$ on $S$. By base change, we may replace $S$ by $\operatorname{Spec} 0_{S, s}$. Then $u$ is étale (Remark 3.20). Thus, it follows from [loc. cit., V.3.7].

Therefore, the pair $\left(\mathscr{C}_{(S, B)}, F_{\bar{S}}\right)$ satisfies Axioms (G1)-(G6) in [loc. cit., V.4], which proves the theorem.

Definition 3.22. Let $(S, B, \bar{s})$ be a pointed connected orbifold. The functor $F_{\bar{S}}$ is pro-representable by a profinite group (see [loc. cit., V.5]). This group is called the fundamental group of $(S, B, \bar{s})$ and denoted by $\pi_{1}(S, B, \bar{s})$. We sometimes omit $\bar{s}$ and denote $\pi_{1}(S, B, \bar{S})$ by $\pi_{1}(S, B)$ for simplicity.

Let $\mathscr{Y}=(S, B, \bar{s})$ and $\mathscr{Y}^{\prime}=\left(S^{\prime}, B^{\prime}, \bar{s}^{\prime}\right)$ be two pointed connected orbifolds. Any pointed orbifold (étale) morphism $u: \mathscr{S}^{\prime} \rightarrow \mathscr{Y}$ induces an (injective) homomorphism $u_{*}: \pi_{1}\left(\mathscr{S}^{\prime}\right) \rightarrow \pi_{1}(\mathscr{S})$ (see [loc. cit., V.6]). Since any connected finite étale $S$-scheme induces a connected finite orbifold étale $(S, B)$-orbifold, we obtain a canonical surjective homomorphism $\phi_{9}: \pi_{1}(\mathscr{Y}) \rightarrow \pi_{1}(S, \bar{s})$. If the regular locus of $S$ is open (e.g., $S$ is excellent), then the singular locus of $S$ is a closed subset of codimension at least two. Thus, Zariski-Nagata purity (Lemma 3.5) shows the following:

Proposition 3.23. Let $\mathscr{Y}=(S, B, \bar{s})$ be a pointed connected orbifold. By $S_{0}$ we denote the regular locus of $S$. Assume that $S_{0}$ is an open subset of $S$. By $u: \mathscr{Y}_{0} \rightarrow \mathscr{Y}$ we denote the pointed orbifold étale morphism induced by the inclusion morphism $S_{0} \rightarrow S$. Then the homomorphism $u_{*}: \pi_{1}\left(\mathscr{Y}_{0}\right) \rightarrow \pi_{1}(\mathscr{S})$ induced by $u$ is an isomorphism. If $\mathscr{S}$ is trivial and regular, then $\phi_{9}$ is an isomorphism and, in particular, $\pi_{1}(\mathscr{S}) \cong \pi_{1}(S)$.

Example 3.24. The homomorphism $\phi_{(S)}: \pi_{1}((S)) \rightarrow \pi_{1}(S)$ is not injective in general, where $(S)$ is the trivial orbifold associated to $S$ and we omit the geometric points. Let $k$ be an algebraically closed field of characteristic zero, $n$ an integer greater than one, and $\zeta$ a primitive $n$-th root of unity. Put $S^{\prime}:=\mathbb{A}_{k}^{2}$. Take the coordinate functions $(x, y)$ of $S^{\prime}$. We define an automorphism $\sigma$ on $S^{\prime}$ by $(x, y) \mapsto$ $(\zeta x, \zeta y)$. Take the quotient $u: S^{\prime} \rightarrow S$ of $\sigma$. The scheme $S$ is normal but not regular. The morphism $u$ ramifies only at the origin $o$ of $S^{\prime}$. Since $\pi_{1}\left(S^{\prime} \backslash\{o\}\right) \cong$ $\pi_{1}\left(S^{\prime}\right) \cong 1$ by Zariski-Nagata purity (Lemma 3.5), we obtain the isomorphisms $\pi_{1}((S)) \cong \mathbb{Z} / n \mathbb{Z}$ and $\pi_{1}(S) \cong 1$. Thus, the homomorphism $\phi_{(S)}$ is not injective.

\section{Homotopy exact sequences}

\section{A. Coverings of fibrations.}

Lemma 4.1. Let $f: X \rightarrow S$ be a quasicompact morphism between locally Noetherian schemes. Assume that $X$ is reduced. Then the following are equivalent: 
(1) $S$ is reduced and $f$ is dominant.

(2) The homomorphism $\mathrm{O}_{S} \rightarrow f_{*} \mathrm{O}_{X}$ associated to $f$ is injective.

Assume that (1) and (2) hold and that $X$ is normal and integral. Then $S$ is integral and the following statements are equivalent:

(3) $S$ is normal and the function field of $S$ is algebraically closed in that of $X$.

(4) $\mathrm{O}_{S}$ is integrally closed in $f_{*} \mathrm{O}_{X}$.

If $f$ is generically separable, then (3) and (4) are equivalent to the following:

(5) $S$ is normal and the generic fiber of $f$ is geometrically integral.

Proof. We may assume that $S$ is affine. Put $R:=\Gamma\left(S, \mathcal{O}_{S}\right)$ and $W:=\Gamma\left(X, \mathscr{O}_{X}\right)$. Since $W$ is reduced and the kernel of the homomorphism $R \rightarrow W$ associated to $f$ is the defining ideal of the closure of $f(X)$, the first equivalence holds. Let us show the other statements. Since $X$ is irreducible and $f$ is dominant, the scheme $S$ is irreducible, which implies that $S$ is integral. By $K$ and $L$ we denote the function fields of $S$ and $X$, respectively. By $K^{\prime}$ and $R^{\prime}$ we denote the algebraic closure of $K$ in $L$ and the integral closure of $R$ in $K^{\prime}$, respectively. Then $R^{\prime}=R$ if and only if $R$ is normal and $K^{\prime}=K$. Since $X$ is normal and integral, the ring $W$ is normal and integral [Liu 2002, 4.1.5], which implies that $R^{\prime}$ is the integral closure of $R$ in $W$. Thus, the second equivalence holds. The last statement follows from [EGA IV 2 1965, 4.6.3].

Lemma 4.2. Let $u: X \rightarrow Y$ and $v: Y \rightarrow Z$ be morphisms between integral schemes. Assume that $v \circ u$ is dominant and generically separable and that $v$ is integral. Then $u$ is dominant and generically separable and $v$ is surjective and generically separable.

Proof. Since $v \circ u$ is dominant and $v$ is closed, the morphism $v$ is surjective. Since $v$ is integral and dominant, the preimage of the generic point of $Z$ under $v$ consists of the generic point of $Y$ [Matsumura 1989, 9.3 (ii)]. Furthermore, since $v \circ u$ is dominant and generically separable, the morphism $u$ is dominant and the morphism $v$ is generically separable. Note the following: for any field extensions $L / K, M / L$, and $N / L$, the ring $M \otimes_{L} N$ may be regarded as a subring of $M \otimes_{K} N$ since $M \otimes_{K} N \cong M \otimes_{L} N \otimes_{L}\left(L \otimes_{K} L\right)$ and $L \cong L \otimes_{K} K \subset L \otimes_{K} L$. Thus, the morphism $u$ is generically separable.

Definition 4.3. Condition $(D)$ on morphisms $f: X \rightarrow S, u^{\prime}: X^{\prime} \rightarrow X, u: S^{\prime} \rightarrow S$, and $f^{\prime}: X^{\prime} \rightarrow S^{\prime}$ between locally Noetherian schemes consists of the following conditions:

(1) $f$ is quasicompact, surjective, and generically separable.

(2) $u^{\prime}$ is finite, surjective, and generically separable. 
(3) $u$ is integral.

(4) The homomorphism $\mathrm{O}_{S} \rightarrow f_{*} \mathrm{O}_{X}$ associated to $f$ is injective and $\mathrm{O}_{S}$ is integrally closed in $f_{*} \mathrm{O}_{X}$.

(5) The homomorphism $\mathrm{O}_{S^{\prime}} \rightarrow f_{*}^{\prime} \mathrm{O}_{X^{\prime}}$ associated to $f^{\prime}$ is injective and $\mathscr{O}_{S^{\prime}}$ is integrally closed in $f_{*}^{\prime} \mathrm{O}_{X^{\prime}}$.

(6) The diagram

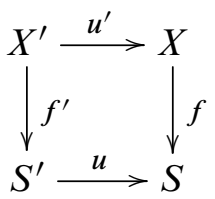

is commutative.

Remark 4.4. Conditions (1)-(3) imply that $f^{\prime}$ is quasicompact. Conditions (1)-(6) imply that $u$ is given by the integral closure of $\mathscr{O}_{S}$ in $\left(f \circ u^{\prime}\right)_{*} \mathcal{O}_{X^{\prime}}$.

Proposition 4.5. Let $f: X \rightarrow S, f^{\prime}: X^{\prime} \rightarrow S^{\prime}, u: S^{\prime} \rightarrow S$, and $u^{\prime}: X^{\prime} \rightarrow X$ be morphisms between locally Noetherian schemes satisfying Condition (D). Suppose that $X$ is normal and that $X^{\prime}$ is connected and normal. Then:

(1) $X, X^{\prime}, S$, and $S^{\prime}$ are normal and integral.

(2) $u$ is finite, surjective, and generically separable.

(3) $f^{\prime}$ is quasicompact, surjective, and generically separable.

Proof. Since $X^{\prime}$ is connected and $u^{\prime}$ is surjective, the scheme $X$ is connected. Lemma 3.3(1) shows that $X$ and $X^{\prime}$ are integral. Thus, Lemma 4.1 shows that $S$ and $S^{\prime}$ are integral and normal. Therefore, Statement (1) holds. Since $f \circ u^{\prime}$ is surjective and generically separable and $u$ is integral, Lemma 4.2 shows that $f^{\prime}$ is dominant and generically separable and $u$ is surjective and generically separable. Thus, Lemma 3.3(2) shows that $u$ is finite. Therefore, Statement (2) holds. Let us show that $f^{\prime}$ is surjective. We may assume that the finite covering $X^{\prime} / X$ is Galois after replacing $X^{\prime}$ and $S^{\prime}$ by finite coverings. The Galois group $G$ of $X^{\prime} / X$ faithfully acts on the finite covering $S^{\prime} / S$ such that $f^{\prime}$ is $G$-equivariant. Since $f^{\prime}\left(X^{\prime}\right)$ is stable under any element of $G$ and the equalities $u\left(f^{\prime}\left(X^{\prime}\right)\right)=f\left(u^{\prime}\left(X^{\prime}\right)\right)=S$ hold, the morphism $f^{\prime}$ is surjective. Thus, Statement (3) holds.

Proposition 4.6. We use the same notation and assumption as in Proposition 4.5. Let $v: T \rightarrow S$ be one of the following morphisms between schemes:

(a) a smooth morphism;

(b) the localization at a point;

(c) the strict Henselization (or the Henselization) of the spectrum of a local ring;

(d) the completion of the spectrum of an excellent local ring at the closed point. 
By $f_{T}: X_{T} \rightarrow T, f_{T}^{\prime}: X_{T}^{\prime} \rightarrow S_{T}^{\prime}, u_{T}: S_{T}^{\prime} \rightarrow T$, and $u_{T}^{\prime}: X_{T}^{\prime} \rightarrow X_{T}$ we denote the base changes of the $S$-morphisms $f, f^{\prime}, u$, and $u^{\prime}$ via $v$, respectively. Take connected components $Z$ and $Z^{\prime}$ of $T$ and $S_{T}^{\prime}$, respectively. Then:

(1) $Z, f_{T}^{-1}(Z), Z^{\prime}$, and $\left(f_{T}^{\prime}\right)^{-1}\left(Z^{\prime}\right)$ are locally Noetherian, normal, and integral.

(2) $f_{T}, f_{T}^{\prime}, u_{T}$, and $u_{T}^{\prime}$ satisfy Condition $(D)$.

(3) If $v$ is surjective, then $u^{\prime}$ is étale if and only if $u_{T}^{\prime}$ is étale.

Proof. By $U$ we denote any of $T, X_{T}, S_{T}^{\prime}$, and $X_{T}^{\prime}$. Then $U$ is locally Noetherian. Since any fiber of $v$ is geometrically regular, the scheme $U$ is normal [Matsumura 1989, 23.9]. The schemes $Z$ and $Z^{\prime}$ are integral (Lemma 3.3(1)). We may assume that $Z=T$ and $f_{T}^{-1}(Z)=X_{T}$. Since $v$ is a dominant flat morphism between integral schemes, Lemma 4.1 implies that $X_{T}$ is integral. In the same way, we may show that $\left(f_{T}^{\prime}\right)^{-1}\left(Z^{\prime}\right)$ is integral. Thus, Statement (1) holds. Let us show Statement (2). We have only to show that $\mathrm{O}_{T}$ and $\mathrm{O}_{S_{T}^{\prime}}$ are integrally closed in $f_{T *} \mathrm{O}_{X_{T}}$ and $f_{T *}^{\prime}{ }^{\mathrm{O}_{X}^{\prime}}$, respectively. Thus, Statement (2) follows from Lemma 4.1. Statement (3) follows from faithfully flat descent for étale morphisms.

Lemma 4.7. Let $u: X \rightarrow Y$ and $v: Y \rightarrow Z$ be morphisms between locally Noetherian schemes. Put $w:=v \circ u$. Assume that $Y$ and $Z$ are normal. Suppose that $u$ is dominant, $v$ is affine, and $w$ is finite, étale, and dominant. Then $u$ and $v$ are finite, étale, and surjective.

Proof. Since $Z$ is normal and $w$ is étale, the scheme $X$ is normal. We may assume that $X, Y$, and $Z$ are integral (Lemma 3.3(1)). Then $u$ and $v$ are finite surjective morphisms between locally Noetherian normal integral schemes. We have only to show that $u$ and $v$ are étale over any point $z$ on $Z$. Since $w$ is finite, étale, and surjective, there exists an étale morphism $t: U \rightarrow Z$ such that $z \in t(U)$ and the restriction of the base change $X_{U} \rightarrow U$ of $w$ via $t$ to any connected component of $X_{U}$ is an isomorphism [Bosch et al. 1990, 2.3.8]. Thus, by faithfully flat descent for étale morphisms, we may assume that $w$ is an isomorphism. Then $u$ and $v$ induce isomorphisms between the function fields of $X, Y$, and $Z$. Since $X, Y$, and $Z$ are normal and integral and $u$ and $v$ are finite, the morphisms $u$ and $v$ are isomorphisms, which implies that $u$ and $v$ are étale.

Proposition 4.8. Let $f: X \rightarrow S$ and $u: S^{\prime} \rightarrow S$ be morphisms between locally Noetherian normal integral schemes. Assume that $f$ is quasicompact, surjective, and generically separable, $u$ is finite, surjective, and generically separable, and $\mathrm{O}_{S}$ is integrally closed in $f_{*} \mathrm{O}_{X}$. Then there exist a locally Noetherian normal integral scheme $X^{\prime}$ and morphisms $f^{\prime}: X^{\prime} \rightarrow S^{\prime}$ and $u^{\prime}: X^{\prime} \rightarrow X$ satisfying the following:

(a) $f, f^{\prime}, u$, and $u^{\prime}$ satisfy Condition $(D)$. 
(b) For any normal integral scheme $Y$ and any dominant morphisms $h: Y \rightarrow S^{\prime}$ and $\xi: Y \rightarrow X$ satisfying $f \circ \xi=u^{\prime} \circ h$, there exists a unique morphism $\xi^{\prime}: Y \rightarrow X^{\prime}$ such that $u^{\prime} \circ \xi^{\prime}=\xi$ and $f^{\prime} \circ \xi^{\prime}=h$.

Furthermore, the following statements hold:

(1) If $\xi$ in (b) is finite and étale, then $u^{\prime}$ and $\xi^{\prime}$ are finite, étale, and surjective.

(2) If $u$ is étale, then $u^{\prime}$ is étale.

(3) If $f$ is separable and of finite type, then the converse of (2) holds.

Proof. By $K, K^{\prime}$, and $L$ we denote the function fields of $S, S^{\prime}$, and $X$, respectively. Put $L^{\prime}:=L \otimes_{K} K^{\prime}$. Since $K$ is algebraically closed in $L$ (Lemma 4.1) and the extension $K^{\prime} / K$ is finite and separable, the ring $L^{\prime}$ is a field. Furthermore, the field $K^{\prime}$ is algebraically closed in $L^{\prime}$ and the extension $L^{\prime} / L$ is finite and separable. Take the normalization $u^{\prime}: X^{\prime} \rightarrow X$ of $X$ in $L^{\prime}$. Since $u^{\prime}$ is finite (Lemma 3.3(2)), the scheme $X^{\prime}$ is locally Noetherian, normal, and integral. Take the unique morphism $f^{\prime}: X^{\prime} \rightarrow S^{\prime}$ such that $f \circ u^{\prime}=u \circ f^{\prime}$. Then $0_{S^{\prime}}$ is integrally closed in $f_{*}^{\prime} \mathcal{O}_{X^{\prime}}$ (Lemma 4.1). Thus, Condition (a) is satisfied. By construction, Condition (b) is satisfied. Statement (1) follows from Lemma 4.7. By $v: Z \rightarrow X$ and $w: Z \rightarrow S^{\prime}$ we denote the base change of $u$ via $f$ and the base change of $f$ via $u$, respectively. Then $v$ is finite, and $Z$ is an integral scheme with function field $L^{\prime}$. Let us show Statement (2). Assume that $u$ is étale. Then $v$ is étale and $Z$ is normal. Thus, the scheme $Z$ is $X$-isomorphic to $X^{\prime}$, which implies that $u^{\prime}$ is étale. Let us show Statement (3). Assume that $f$ is separable and of finite type and that $u^{\prime}$ is étale. Replacing $X$ by the smooth locus of $f$, we may assume that $f$ is smooth ([Bosch et al. 1990, 2.2.16] and Lemma 4.1). Then $Z$ is normal. Thus, the scheme $Z$ is $X$-isomorphic to $X^{\prime}$, which implies that $v$ is étale. Therefore, Statement (3) follows from faithfully flat descent for étale morphisms.

\section{B. Base spaces of local étale coverings.}

Definition 4.9. Let $R$ be a strictly Henselian Noetherian normal local ring with field of fractions $K$. Take a separable closure $\bar{K}$ of $K$. Put $S:=\operatorname{Spec} R$. Let $f: X \rightarrow S$ be a surjective generically separable morphism between connected Noetherian normal schemes. Assume that $O_{S}$ is integrally closed in $f_{*} \mathscr{O}_{X}$. We define the maximal base field $\tilde{K}$ (of étale coverings of the total space) of $f$ in the following way. Let $\xi: Y \rightarrow X$ be a connected étale covering space. Then $Y$ is normal. Take the normalization

$$
Y \stackrel{h}{\longrightarrow} S^{\prime} \stackrel{u}{\longrightarrow} S
$$

of $S$ in the composite $f \circ \xi: Y \rightarrow X \rightarrow S$. By $K_{\xi}$ we denote the function field of $S^{\prime}$. Then $u$ induces a finite separable field extension $K_{\xi} / K$ (Proposition 4.5). 
We define $\widetilde{K}$ as the composite field of all $K$-embeddings of the finite separable extensions $K_{\xi} / K$ in $\bar{K}$ for all connected étale covering spaces $\xi: Y \rightarrow X$ of $X$.

Remark 4.10. By definition, the field extension $\tilde{K} / K$ is algebraic and Galois.

We use the notation introduced in Definition 4.9.

Proposition 4.11. Let $g: X^{\prime} \rightarrow X$ be a proper birational morphism between regular integral schemes. Then the maximal base field of $f \circ g$ in $\bar{K}$ is equal to $\tilde{K}$.

Proof. By Zariski-Nagata purity (Lemma 3.5; see also [SGA 1 1971, X.3.3]), the base change of finite étale $X$-schemes via $g$ induces an equivalence of categories between the category of finite étale $X$-schemes and the category of finite étale $X^{\prime}$-schemes, which proves the proposition.

Lemma 4.12. Let $L / K$ be a finite field extension in $\bar{K}$. Then $L \subset \widetilde{K}$ if and only if there exists a connected étale covering space of $X$ that induces the extension $L / K$. Proof. The "if" part follows from the definition of $\widetilde{K}$. Since any finite fiber product of étale covering spaces of $X$ over $X$ is an étale covering space of $X$, the "only if" part follows from Proposition 4.8(1).

By $k$ we denote the residue field of $R$. By $\left\{Z_{i}\right\}_{i \in I}$ we denote the set of all irreducible components of the special fiber $X_{k}$ of $f$ with the reduced structures. Take the integral closure $k_{i}$ of $k$ in $\Gamma\left(Z_{i}, O_{Z_{i}}\right)$.

Lemma 4.13. The ring $k_{i}$ is a field. If $f$ is of finite type, then the field extension $k_{i} / k$ is finite and purely inseparable for any $i \in I$.

Proof. Since $k_{i}$ is an integral extension of the field $k$, the integral domain $k_{i}$ is a field. Assume that $f$ is of finite type. Since $Z_{i}$ is finite type over $k$, the function field $K_{i}$ of $Z_{i}$ is finitely generated over $k$. Since $k_{i} \subset K_{i}$ and $k$ is separably closed, the last statement holds.

Suppose that $R$ is a discrete valuation ring. Then $f$ is flat. The closed subscheme $X_{k}$ is a divisor on $X$ and, for any $i \in I$, the closed subscheme $Z_{i}$ is a prime divisor on $X$ (Lemma 3.4(2)). We may write $X_{k}=\sum_{i \in I} m_{i} Z_{i}$.

Lemma 4.14. Suppose that $R$ is a discrete valuation ring. Assume that $f$ is of finite type. Put $n_{i}:=\left[k_{i}: k\right]$, which is finite by Lemma 4.13. Let $\xi: Y \rightarrow X$ be a connected étale covering space. Take the normalization

$$
Y \stackrel{h}{\longrightarrow} S^{\prime} \stackrel{u}{\longrightarrow} S
$$

of $S$ in the composite $f \circ \xi: Y \rightarrow X \rightarrow S$. Then the degree of $u$ divides $\operatorname{gcd}\left(m_{i}\right)_{i \in I} \cdot \operatorname{gcd}\left(n_{i}\right)_{i \in I}$. 
Proof. By $m$ and $n$ we denote the ramification index of $S^{\prime} / S$ and the degree of the residue field extension of $S^{\prime} / S$, respectively. Then $m \mid m_{i}$ and $n \mid n_{i}$ for any $i \in I$ since $\xi$ is étale and $k$ is separably closed. Since $R$ is a Henselian discrete valuation ring, the degree of $u$ is equal to $m n$, which concludes the proof.

Proposition 4.15. We use the notation introduced in Definition 4.9. By $k$ we denote the residue field of $R$. Suppose that $R$ is a discrete valuation ring. Assume that $f$ is of finite type. Then:

(1) The field extension $\widetilde{K} / K$ is finite and Galois.

(2) If $f$ is separable, then $\widetilde{K}=K$.

(3) If $k$ is perfect, then $[\widetilde{K}: K]$ divides the multiplicity of the special fiber of $f$ (Section 2).

(4) If $f$ is proper and a finite field extension $K^{\prime} / K$ in $\bar{K}$ satisfies $X\left(K^{\prime}\right) \neq \varnothing$, then $\widetilde{K} \subset K^{\prime}$.

Proof. Statement (1) (resp. (2) and (3)) follows from Lemma 4.14 since $\widetilde{K} / K$ is Galois (Remark 4.10) (resp. $m_{i}=1$ and $k_{i}=k$ for any $i \in I$, and $k_{i}=k$ for any $i \in I$ (Lemma 4.13)). Let us show Statement (4). By Proposition 4.8(1), we may assume that $\widetilde{K} \cap K^{\prime}=K$. By $S^{\prime}$ and $\widetilde{S}$ we denote the normalizations of $S$ in $K^{\prime}$ and $\widetilde{K}$, respectively. Take the scheme $X^{\prime}$ (resp. $\tilde{X}$ ) and the morphism $u^{\prime}: X^{\prime} \rightarrow X$ (resp. $\tilde{u}: \tilde{X} \rightarrow X$ and $\tilde{f}: \tilde{X} \rightarrow \widetilde{S}$ ) given by Proposition 4.8. Then $\tilde{u}: \tilde{X} \rightarrow X$ is an étale covering space. Since the base change of $\tilde{u}$ via $u^{\prime}$ induces $\tilde{K} K^{\prime} / K^{\prime}$, we have only to show that $\widetilde{K}=K$ whenever $X(K) \neq \varnothing$. Assume that $X(K) \neq \varnothing$. Then $f$ admits a section by the valuative criterion for properness. Since the pullback of any section of $f$ via $\tilde{u}$ induces a section of $\tilde{f}$ and $S$ is strictly Henselian, the degree of $\tilde{u}$ is equal to 1 , which concludes the proof.

Example 4.16. Let us give an example of a morphism $f: X \rightarrow S$ of finite type with $[\widetilde{K}: K]=\infty$ when $\operatorname{dim} S>1$. Assume that $k$ is algebraically closed, the characteristic of $k$ is not equal to 3 , and $R=k \llbracket x, y, z \rrbracket /\left(x^{3}+y^{3}+z^{3}\right)$. By $s$ we denote the closed point of $S$. Put $S_{0}:=S \backslash\{s\}$. Then $\pi_{1}((S)) \cong \pi_{1}\left(S_{0}\right)$ (Proposition 3.23). Take the blowing-up $f: X \rightarrow S$ of $S$ at $s$. Then $X$ is regular. Put $E:=f^{-1}(s)$. The reduction $E_{\text {red }}$ of $E$ is $k$-isomorphic to an elliptic curve over $k$ and the multiplicity of $E$ is equal to 3. The morphism $f$ is not flat at any point on $E$ (Lemma 3.4(2)). The inclusion morphisms $S_{0} \rightarrow X, E_{\mathrm{red}} \rightarrow E$, and $E \rightarrow X$ induce a surjective homomorphism $\pi_{1}\left(S_{0}\right) \rightarrow \pi_{1}(X)$, an injective homomorphism $\pi_{1}\left(E_{\text {red }}\right) \rightarrow \pi_{1}(E)$, and an isomorphism $\pi_{1}(E) \cong \pi_{1}(X)$, respectively [SGA $4 \frac{1}{2}$ 1977, IV.2.2]. Since $\pi_{1}\left(E_{\text {red }}\right)$ is not finite, the extension $\widetilde{K} / K$ is infinite.

4C. Homotopy exact sequences. In this subsection, we give homotopy exact sequences for fibrations satisfying the following conditions: 
Definition 4.17. Condition $(C)$ on a triple $(X, S, f)$ consists of the following conditions:

(1) $X$ and $S$ are locally Noetherian normal integral schemes.

(2) $f: X \rightarrow S$ is a surjective morphism of finite type.

(3) $\mathrm{O}_{S}$ is integrally closed in $f_{*} \mathrm{O}_{X}$ (Conditions (1) and (2) imply that the homomorphism $\mathrm{O}_{S} \rightarrow f_{*} \mathrm{O}_{X}$ associated to $f$ is injective (Lemma 4.1)).

(4) The geometric generic fiber of $f$ is reduced.

Condition $\left(C^{*}\right)$ on a triple $(X, S, f)$ is Condition $(\mathrm{C})$ and the following conditions:

(5) $X$ is regular.

(6) $f$ is flat in codimension one.

Remark 4.18. In the case where $f$ is proper, Conditions (2) and (3) are equivalent to the following conditions:

$\left(2^{\prime}\right) f: X \rightarrow S$ is proper.

(3') The homomorphism $\mathrm{O}_{S} \rightarrow f_{*} \mathrm{O}_{X}$ associated to $f$ is an isomorphism.

Remark 4.19. In our studies on homotopy exact sequences, Condition $\left(C^{*}\right)$ is necessary. This condition is used to describe the effect of the nonreduced geometric fibers of $f$ on étale covering spaces of $X$ in terms of an orbifold $(S, B)$. Conditions (1)-(3) are used to produce a finite covering space of $S$ by taking the normalization of $S$ in the composite of a finite covering map of $X$ and $f$. In the case where $f$ is proper, this normalization may be given by the Stein factorization of the composite. Condition (5) is used to apply Zariski-Nagata purity (Lemma 3.5) to a finite covering space of $X$. In particular, the condition that the finite covering map is étale may be checked in codimension one. Condition (4) enables Condition (6) to encode this condition as the data of ramifications $B$ of an orbifold $(S, B)$. See Theorem 4.22 and Remark 4.26 for Condition (C).

Example 4.20. Take $k, n, u: S^{\prime} \rightarrow S$, and $\sigma$ as in Example 3.24. Let $E$ be an elliptic curve over $k$. Put $X^{\prime}:=E \times_{k} S^{\prime}$. By $f^{\prime}: X^{\prime} \rightarrow S^{\prime}$ we denote the second projection. Choose a primitive $n$-torsion point $P$ on $E$. We define an action $\tau$ on $X^{\prime}$ as the product of the translation by the addition of $P$ on $E$ and the action of $\sigma$ on $S^{\prime}$. We take the quotient $u^{\prime}: X^{\prime} \rightarrow X$ of $\tau$. Since $f^{\prime}$ is equivariant with respect to $\tau$ and $\sigma$, we obtain a morphism $f: X \rightarrow S$. The triple $(X, S, f)$ satisfies Condition (C). The morphisms $f, f^{\prime}, u$, and $u^{\prime}$ satisfy Condition (D) (Definition 4.3). Since $X$ is regular and $S$ is not regular, the morphism $f$ is not flat [Matsumura 1989, 23.7 (i)]. However, the morphism $f$ is flat in codimension one since $f$ is flat over the regular locus of $S$ [Matsumura 1989, 23.1]. In particular, the triple $(X, S, f)$ satisfies Condition $\left(\mathrm{C}^{*}\right)$. 
Example 4.21. Take $k, u: S^{\prime} \rightarrow S$, and $o$ as in Example 3.24. Take the blowing-ups $f: X \rightarrow S$ and $f^{\prime}: X^{\prime} \rightarrow S^{\prime}$ of $S$ and $S^{\prime}$ at $u(o)$ and $o$, respectively. Then $X$ and $X^{\prime}$ are regular. The universal property of blowing-up shows that there exists a unique morphism $u^{\prime}: X^{\prime} \rightarrow X$ such that $f \circ u^{\prime}=u \circ f^{\prime}$. The morphism $u^{\prime}$ ramifies along the exceptional divisor of $f^{\prime}$. The morphisms $f, f^{\prime}, u$, and $u^{\prime}$ satisfy Condition (D) (Definition 4.3). The triple $(X, S, f)$ satisfies Condition (C). However, the morphism $f$ is not flat in codimension one (Lemma 3.4(2)). In particular, the triple $(X, S, f)$ does not satisfy Condition $\left(\mathrm{C}^{*}\right)$.

We first generalize Grothendieck's homotopy exact sequence to the case where fibrations are not necessarily proper:

Theorem 4.22. Let $(X, S, f)$ be a triple satisfying Condition $(C)$ (Definition 4.17). Assume that $f$ is separable. Choose a connected geometric fiber $i: X_{0} \rightarrow X$ of $f$ (e.g., the geometric generic fiber of $f$ ). Take a geometric point $\bar{x}_{0}$ on $X_{0}$. Put $\bar{x}:=$ $i\left(\bar{x}_{0}\right)$ and $\bar{s}:=f(\bar{x})$. The morphisms $i$ and $f$ induce canonical homomorphisms $i_{*}: \pi_{1}\left(X_{0}, \bar{x}_{0}\right) \rightarrow \pi_{1}(X, \bar{x})$ and $f_{*}: \pi_{1}(X, \bar{x}) \rightarrow \pi_{1}(S, \bar{s})$, respectively. Then the sequence

$$
\pi_{1}\left(X_{0}, \bar{x}_{0}\right) \stackrel{i_{*}}{\longrightarrow} \pi_{1}(X, \bar{x}) \stackrel{f_{*}}{\longrightarrow} \pi_{1}(S, \bar{s}) \longrightarrow 1
$$

is exact.

Proof. We have only to show the exactness at $\pi_{1}(X)$. Since $X_{0}$ is a geometric fiber of $f$, the relation $\operatorname{Im} i_{*} \subset \operatorname{Ker} f_{*}$ holds. Let us show that $\operatorname{Ker} f_{*} \subset \operatorname{Im} i_{*}$. Take $\eta \in \operatorname{Ker} f_{*}$. We have only to show the following: for any connected Galois étale covering space $\xi: Y \rightarrow X$, the element $\eta$ acts trivially on $\pi_{0}\left(\xi^{-1}\left(X_{0}\right)\right)$, where we denote the base change of $X_{0}$ via $\xi$ by $\xi^{-1}\left(X_{0}\right)$. Take the normalization

$$
Y \stackrel{h}{\longrightarrow} S^{\prime} \stackrel{u}{\longrightarrow} S
$$

of $S$ in the composite $f \circ \xi: Y \rightarrow X \rightarrow S$. Then $u: S^{\prime} \rightarrow S$ is an étale covering space (Proposition 4.5(2) and Proposition 4.8(3)). The action of $\eta$ on $Y / X$ induces an action of $\eta$ on $S^{\prime} / S$. Since $\eta \in \operatorname{Ker} f_{*}$, the element $\eta$ acts trivially on $S^{\prime} / S$. Thus, the element $\eta$ acts trivially on $\pi_{0}\left(\xi^{-1}\left(X_{0}\right)\right)$, which implies that $\eta \in \operatorname{Im} i_{*}$. Therefore, the sequence is exact.

Definition 4.23. Let $(X, S, f)$ be a triple satisfying Condition (C) (Definition 4.17). We define the orbifold $(S, B)$ associated to $f$ in the following way. By $P(S)$ we denote the set of all points on $S$ of codimension one. Take $s \in P(S)$. Put $K_{s}:=$ Frac $\mathcal{O}_{S, s}^{\text {sh }}$. By $f_{s}$ we denote the base change of $f$ via the composite Spec $\mathbb{O}_{S, s}^{\text {sh }} \rightarrow$ Spec $\mathrm{O}_{S, s} \rightarrow S$ of the canonical morphisms. Take the maximal base field $\widetilde{K}_{S}$ of $f_{S}$ (Definition 4.9). Proposition 4.15(1) shows that the field extension $\widetilde{K_{S}} / K_{S}$ is finite and Galois. We define a map $B$ on $P(S)$ by $s \mapsto \widetilde{K_{S}} / K_{S}$ (Definition 3.6). Let us show that the pair $(S, B)$ is an orbifold. By $S_{0}$ we denote the open subscheme of $S$ 
that is the complement of the closure of Supp $B$. Take a nonempty open subscheme $S_{1}$ of $S$ over which $f$ is separable. Then $S_{1} \subset S_{0}$ (Proposition 4.15(2)), which implies that $\operatorname{Supp} B$ is locally finite. Thus, the pair $(S, B)$ is an orbifold.

Using the above orbifold, we give an étaleness criterion for finite coverings of $X$ :

Theorem 4.24. Let $(X, S, f)$ be a triple satisfying Condition $\left(C^{*}\right)$ (Definition 4.17). Take the orbifold $(S, B)$ associated to $f$ (Definition 4.23). Let $u: S^{\prime} \rightarrow S$ be a finite surjective generically separable morphism between locally Noetherian normal integral schemes. Take the scheme $X^{\prime}$ and the morphism $u^{\prime}: X^{\prime} \rightarrow X$ given by Proposition 4.8. Then $u^{\prime}$ is étale if and only if $u$ induces an orbifold étale morphism $\left(S^{\prime}, B^{\prime}\right) \rightarrow(S, B)$ (Definition 3.12).

Proof. First, we assume that $S=\operatorname{Spec} Q$ and $S^{\prime}=\operatorname{Spec} Q^{\prime}$ where $Q$ and $Q^{\prime}$ are discrete valuation rings. The morphism $u$ induces a finite flat extension $Q^{\prime} / Q$ of discrete valuation rings. Put $J:=$ Frac $Q, J^{\prime}:=$ Frac $Q^{\prime}, K:=$ Frac $Q^{\text {sh }}$, and $K^{\prime}:=J^{\prime} \otimes_{J} K$. The field extension $J^{\prime} / J$ induced by $u$ is finite and separable. Take the maximal unramified extension $I$ of $J$ in $J^{\prime}$. We may embed $I$ in $K$ over $J$. By (1) and (2) of Proposition 4.8, we may assume that $J=I$. Then $K^{\prime}$ is a field. By Proposition 4.6, we may assume that $J=K$ and $J^{\prime}=K^{\prime}$. Then the theorem follows from Lemma 4.12.

Next, let us show the general case. The "only if" part follows from the first case and Proposition 4.6. Let us show the "if" part. Since $f$ maps any point of codimension one to a point of codimension at most one (Lemma 3.4(2)), the first case and Proposition 4.6 show that $u^{\prime}$ is étale in codimension one. Thus, ZariskiNagata purity (Lemma 3.5) shows that $u^{\prime}$ is étale, which proves the "if" part.

Definition 4.25. Let $(X, S, f)$ be a triple satisfying Condition $\left(\mathrm{C}^{*}\right)$ (Definition 4.17). Take the orbifold $(S, B)$ associated to $f$ (Definition 4.23). Choose a geometric point $\bar{x}$ on $X$. Put $\bar{s}:=f(\bar{x})$. Assume that the image of $\bar{s}$ on $S$ is a regular point on $S$ (e.g., the generic point of $S$ ). We define the homomorphism $f_{*}^{\text {orb }}: \pi_{1}(X, \bar{x}) \rightarrow$ $\pi_{1}(S, B, \bar{s})$ induced by $f$ in the following way. Let $u:\left(S^{\prime}, B^{\prime}\right) \rightarrow(S, B)$ be a connected orbifold étale covering space. Take the scheme $X^{\prime}$ and the morphism $u^{\prime}: X^{\prime} \rightarrow X$ given by Proposition 4.8. Then $u^{\prime}: X^{\prime} \rightarrow X$ is a connected étale covering space (Theorem 4.24). Thus, we obtain a surjective homomorphism $f_{*}^{\text {orb }}: \pi_{1}(X, \bar{x}) \rightarrow \pi_{1}(S, B, \bar{s})$.

Proof of Theorem 1.1. We may show the theorem in the same way as in the proof of Theorem 4.22. We have only to use Theorem 4.24 instead of Proposition 4.8(3).

Remark 4.26. We generalize the definition of an orbifold $(S, B)$ (Definition 3.6) by the following two modifications: replace $P(S)$ by all points on $S$; remove the finiteness assumption on $B_{S} / K_{S}$. We may define the fundamental group of $(S, B)$ in the same way as in the case of an orbifold. The morphism $f$ induces 
a generalized orbifold $(S, B)$. Note that if $f$ is separable, then $(S, B)$ is trivial (Proposition 4.8(3)). Replacing an orbifold by a generalized orbifold, we may show Theorems 4.24 and 1.1 for any triple $(X, S, f)$ satisfying Condition (C) (Definition 4.17) without the regularity assumption on $X$ and the flatness assumption on $f$ in codimension one (Examples 4.16 and 4.21).

\section{Orbifold trivializations of orbifold curves}

In this section, we fix an algebraically closed field $k$ of characteristic $p \geq 0$. We study orbifold trivializations of orbifold $k$-curves and classify simply connected cyclic orbifold $k$-curves.

Definition 5.1. An orbifold $(C, B)$ (Definition 3.6) is called an orbifold $k$-curve (resp. a proper orbifold $k$-curve) if $C$ is a $k$-curve (resp. a proper $k$-curve). If $p>0$ and $\left[B_{s}: K_{s}\right]$ is power of $p$ for any $s \in P(C)$, we say that an orbifold $(C, B)$ is a p-orbifold $k$-curve.

Since the underlying scheme of any orbifold $k$-curve is a smooth $k$-curve, we study ramified coverings of smooth $k$-curves. The Riemann-Hurwitz formula shows the following:

Lemma 5.2. Let $u: C \rightarrow \mathbb{P}_{k}^{1}$ be a finite tamely ramified $k$-morphism of degree $d$ between connected proper smooth $k$-curves. By $N$ we denote the number of the branched points of $u$. Then:

(1) $N \neq 1$.

(2) If $N=2$, then $C$ is isomorphic to $\mathbb{P}_{k}^{1}$, u ramifies at exactly two points, and both of the two ramification indices are equal to $d$.

Proposition 5.3. Let $\left(\mathbb{P}_{k}^{1}, B\right)$ be a proper tame orbifold $k$-curve. If \# Supp $B \leq 1$, then $\left(\mathbb{P}_{k}^{1}, B\right)$ is simply connected. Assume that $\operatorname{Supp} B=\{0, \infty\}$. For $s=0$ and $\infty$ we put $n_{s}:=\left[B_{s}: K_{S}\right]$. Then:

(1) The orbifold $\left(\mathbb{P}_{k}^{1}, B\right)$ is simply connected if and only if $\operatorname{gcd}\left(n_{0}, n_{\infty}\right)=1$.

(2) There exists an orbifold trivialization of $\left(\mathbb{P}_{k}^{1}, B\right)$ if and only if $n_{0}=n_{\infty}$.

In Statement (2), the restriction of the orbifold trivialization to any connected component ramifies at exactly two points.

Proof. The first statement follows from Lemma 5.2(1). Let us show the other statements. Put $d:=\operatorname{gcd}\left(n_{0}, n_{\infty}\right)$. Take a parameter $t$ of $\mathbb{P}_{k}^{1}$ so that $t(0)=0$ and $t(\infty)=\infty$. Then the $k$-morphism $\mathbb{P}_{k}^{1} \rightarrow \mathbb{P}_{k}^{1}, t \mapsto t^{d}$ induces an orbifold étale morphism $\left(\mathbb{P}_{k}^{1}, B^{\prime}\right) \rightarrow\left(\mathbb{P}_{k}^{1}, B\right)$, where the equalities

$$
\left[B_{s}^{\prime}: K_{s}\right]= \begin{cases}n_{i} / d & \text { if } s=0 \text { or } \infty \\ 1 & \text { otherwise }\end{cases}
$$


hold. Thus, Lemma 5.2 proves the proposition.

Proposition 5.4. Let $\left(\mathbb{P}_{k}^{1}, B\right)$ be a proper tame orbifold $k$-curve with $\operatorname{Supp} B=$ $\{0,1, \infty\}$. For $s=0,1$, and $\infty$, we put $n_{s}:=\left[B_{s}: K_{s}\right]$. Assume that $n_{0}, n_{1}$, and $n_{\infty}$ are pairwise coprime. Then there exists a Galois orbifold trivialization of $\left(\mathbb{P}_{k}^{1}, B\right)$ with noncommutative simple Galois group.

Proof. First, we consider the case $p=0$. By $F_{2}=\left\langle x_{0}, y_{0}\right\rangle$ we denote the free group of rank two. Take the elements $x, y$, and $z$ of the triangle group $\Delta\left(n_{0}, n_{1}, n_{\infty}\right)$ in the definition of $\Delta\left(n_{0}, n_{1}, n_{\infty}\right)$ in Section A. We define a homomorphism $\phi: F_{2} \rightarrow$ $\Delta\left(n_{0}, n_{1}, n_{\infty}\right)$ by $x_{0} \mapsto x$ and $y_{0} \mapsto y$. Then the equality $\phi\left(y_{0}^{-1} x_{0}^{-1}\right)=z$ holds. Since the étale fundamental group of $\mathbb{P}_{k}^{1} \backslash\{0,1, \infty\}$ is isomorphic to the profinite completion of $F_{2}$ (see [Lieblich and Olsson 2010] for a purely algebraic proof), Theorem A.9 shows the proposition.

Next, we consider the case $p>0$. Take a complete discrete valuation ring $R$ of characteristic zero whose residue field is isomorphic to $k$ (e.g., the ring of Witt vectors over $k$ ). Put $K:=$ Frac $R$. The case $p=0$ shows the following: replacing $R$ by a finite extension of $R$, there exist a finite noncommutative simple group $G$ and a $K$-morphism $w_{K}: Y_{K} \rightarrow \mathbb{P}_{K}^{1}$ between connected proper smooth $K$-curves satisfying the following condition:

(0) $w_{K}$ is the quotient morphism $Y_{K} \rightarrow \mathbb{P}_{K}^{1}=Y_{K} / G$ whose branch points are equal to 0,1 , and $\infty$, over which each ramification index is equal to $n_{0}, n_{1}$, and $n_{\infty}$, respectively.

In the following, we take an appropriate $R$-model $w$ of $w_{K}$ in the same way as in [Raynaud 1994, §§6.1-6.3]. Remark that in [Raynaud 1994, §6] the group $G$ is a quasi- $p$-group and the ramification indices of $w_{K}$ are equal to powers of $p$. However, these conditions are used only from Lemma 6.3.6. Replacing $R$ by a finite extension of $R$, we obtain an $R$-morphism $w: Y \rightarrow P$ between $R$-schemes satisfying Conditions (1)-(6):

(1) $Y$ is a projective normal semistable $R$-curve that is an $R$-model of $Y_{K}$.

(2) $P$ is a projective normal semistable $R$-curve that is an $R$-model of $\mathbb{P}_{K}^{1}$.

(3) $w$ is the quotient morphism $Y \rightarrow P=Y / G$.

(4) The restriction of $w$ to the generic fibers is equal to $w_{K}$.

(5) The closure of each branch point of $w_{K}$ in $P$ is contained in the smooth locus $P_{\text {sm }}$ of the $R$-scheme $P$.

To state Condition (6) below, we introduce notation. Condition (2) shows the following two statements on the special fiber $P_{k}$ of $P$ : any irreducible component is isomorphic to $\mathbb{P}_{k}^{1}$, and the dual graph of the irreducible components is a tree $\Gamma_{P}$. By $0_{R}, 1_{R}$, and $\infty_{R}$ we denote the closures of 0,1 , and $\infty$ on $\mathbb{P}_{K}^{1}$ in $P$, respectively. 
For $s=0,1$, and $\infty$, we denote the reduction of $s_{R}$ by $s_{k}$. Condition (5) implies that $s_{k}$ is contained in exactly one irreducible component $C_{s}$. We denote the vertex of $\Gamma_{P}$ corresponding to $C_{s}$ by $e_{s}$. Any two vertices $e_{s}$ and $e_{t}$ are connected by a unique line $l_{s t}$ on $\Gamma_{P}$. The intersection $l_{01} \cap l_{1 \infty} \cap l_{\infty 0}$ is exactly one vertex $e_{01 \infty}$. We denote the irreducible component corresponding to $e_{01 \infty}$ by $C_{01 \infty}$.

(6) $P$ is a successive blowing-up of $\mathbb{P}_{R}^{1}$, and the strict transform of the special fiber of $\mathbb{P}_{R}^{1}$ is equal to $C_{01 \infty}$.

Take a subgroup $H$ of $G$. By Condition (1), we obtain an $R$-morphism $u: Y \rightarrow X$ between $R$-schemes satisfying Conditions (7) and (8) [Raynaud 1990, Corollaire of Proposition 5]:

(7) $X$ is a proper normal semistable $R$-curve with connected smooth generic fiber.

(8) $u$ is the quotient morphism $Y \rightarrow X=Y / H$.

Conditions (3) and (8) give an $R$-morphism $v: X \rightarrow P$ between $R$-schemes such that $w=v \circ u$. Furthermore, the following condition is satisfied:

(9) $u, v$, and $w$ are finite and surjective.

Since $v$ and $w$ are finite (Condition (9)), $X$ and $Y$ are Cohen-Macaulay (Conditions (1) and (7) and [EGA IV 2 1965, 5.8.6]), and $P_{\text {sm }}$ is regular (Condition (2)), the following condition is satisfied [Matsumura 1989, 23.1]:

(10) $v$ and $w$ are flat over $P_{\mathrm{sm}}$.

By $\Gamma_{Y}$ and $\Gamma_{X}$ we denote the dual graphs of the irreducible components of the special fibers $Y_{k}$ and $X_{k}$ of $Y$ and $X$. The group $G$ acts on $\Gamma_{Y}$. The quotient morphisms $u$ and $v$ induce the quotient maps of the actions of $G$ and $H$ from the vertices of $\Gamma_{Y}$ to the vertices of $\Gamma_{P}$ and $\Gamma_{X}$, respectively. If an element $g$ of $G$ fixes an edge $e$ of $\Gamma_{Y}$, then $g$ does not exchange the two vertices on the edge $e$ [Raynaud 1994, 6.3.5]. Thus, the quotients of the actions of $G$ and $H$ on $\Gamma_{Y}$ are canonically isomorphic to $\Gamma_{P}$ and $\Gamma_{X}$, respectively. Therefore, the morphisms $u$ and $v$ induce maps $\Gamma_{Y} \rightarrow \Gamma_{X} \rightarrow \Gamma_{P}$, respectively, that preserve the vertices and the edges.

Take the generic point $\lambda$ of an irreducible component of $P_{k}$. Choose the generic point $\eta$ of an irreducible component of $Y_{k}$ over $\lambda$. Conditions (9) and (10) show that $\mathrm{O}_{Y, \eta} / \mathcal{O}_{P, \lambda}$ is a finite flat extension of discrete valuation rings. Conditions (1) and (2) imply that the ramification index of $\mathscr{O}_{Y, \eta} / \mathscr{O}_{P, \lambda}$ is equal to 1 . Thus, the inertia group $I_{\eta}$ of $\mathrm{O}_{Y, \eta} / \mathcal{O}_{P, \lambda}$ is a $p$-group. Since $G$ is a noncommutative simple group and any simple $p$-group is commutative, the inequality $G \neq I_{\eta}$ holds. We take the above subgroup $H$ of $G$ so that $H=I_{\eta}$. Put $\theta:=u(\eta)$. Then the extension of the residue fields of $\mathscr{O}_{X, \theta} / \mathscr{O}_{P, \lambda}$ is separable. Conditions (2) and (7) imply that the ramification index of $\mathscr{O}_{X, \theta} / \mathscr{O}_{P, \lambda}$ is equal to 1 . Thus, the extension $\mathscr{O}_{X, \theta} / \mathcal{O}_{P, \lambda}$ is étale. 
Take $g \in G$. Replacing $\eta$ and $H$ by $g \eta$ and $g H g^{-1}$, respectively, we obtain $X^{g}$ and $\theta^{g}$ corresponding to $X$ and $\theta$, respectively, in the same way as in the above argument. Furthermore, the extension $\mathscr{O}_{X^{g}, \theta^{g}} / \mathscr{O}_{P, \lambda}$ is étale. Since $v$ is finite (Condition (9)) and $R$ is excellent, the normalization $Y^{\prime}$ of the fiber product of $X^{g}$ for all $g \in G$ over $P$ is finite over $P$. By $Y_{K}^{\prime}$ we denote the generic fiber of $Y^{\prime}$. Since $G$ is simple and $G \neq H$, the intersection $\bigcap_{g \in G} g H g^{-1}$ is the trivial group. Thus, any connected component of $Y_{K}^{\prime}$ is $P_{K}$-isomorphic to $Y_{K}$ (Lemma 3.14). Since $Y$ is normal (Condition (1)) and $w$ is finite (Condition (9)), any connected component of $Y^{\prime}$ is $P$-isomorphic to $Y$. Thus, the product of $\theta^{g}$ for all $g \in G$ over $\lambda$ gives a point $\eta_{0}$ on $Y$ over $\lambda$ such that the extension ${ }_{Y}, \eta_{0} / \mathscr{O}_{P, \lambda}$ is étale. Since $w$ is a Galois covering, the extension $0_{Y, g \eta_{0}} / \mathscr{O}_{P, \lambda}$ is étale for any $g \in G$. Since $\lambda$ is arbitrary, the morphism $w$ is étale at the generic point of any irreducible component of $Y_{k}$. Put $P^{\prime}:=P_{\mathrm{sm}} \backslash\left(0_{R} \cup 1_{R} \cup \infty_{R}\right)$. By $w^{\prime}$ we denote the restriction $\left.w\right|_{w^{-1}\left(P^{\prime}\right)}: w^{-1}\left(P^{\prime}\right) \rightarrow P^{\prime}$. Condition (0) implies that the restriction of $w^{\prime}$ to the generic fibers is étale. Thus, the morphism $w^{\prime}$ is étale in codimension one. Therefore, Zariski-Nagata purity (Lemma 3.5) shows that $w^{\prime}$ is étale. Thus, by Lemma 5.2 and the same method as above, we may assume that $\Gamma_{P}=l_{01} \cup l_{1 \infty} \cup l_{\infty 0}$ after successive blowing-down of exceptional curves on $Y$ and $P$ (Condition (6)).

The normalizations of the preimages of $C_{s}$ and $C_{01 \infty}$ under $w$ are proper smooth $k$-curves $C_{s}^{\prime}$ and $C_{01 \infty}^{\prime}$, respectively. The covering $C_{s}^{\prime} / C_{s}$ branches at $s_{k}$, over which each ramification index is equal to $n_{s}$ ([Raynaud 1994, 6.3.2] and Conditions (0), (4), and (5)). Since $w^{\prime}$ is étale, Lemma 5.2 implies that the preimage $C_{01 \infty}^{\prime}$ is connected and the covering $C_{01 \infty}^{\prime} / C_{01 \infty}$ branches at exactly three points, over which each ramification index is equal to $n_{0}, n_{1}$, and $n_{\infty}$, respectively. Therefore, the covering $C_{01 \infty}^{\prime} / C_{01 \infty}$ induces a desired orbifold trivialization.

Lemma 5.5. Let $C$ be a connected proper smooth $k$-curve of positive genus. Take a closed point $s$ on $C$ and an integer $n$. Assume that $p \nmid n$. Then there exists a connected étale covering space $u: C^{\prime} \rightarrow C$, a divisor $D$ on $C^{\prime}$, and a rational function $h$ on $C^{\prime}$ such that $u^{*}[s]-n D=(h)$, where $[s]$ and $(h)$ are the divisors defined by $s$ and $h$, respectively.

Proof. We may assume that $n$ is positive. Since the genus of $C$ is positive and $p \nmid n$, we may take a connected étale covering space $u: C^{\prime} \rightarrow C$ of degree $n$. We denote the genus of $C^{\prime}$ by $g$. By $J$ we denote the Jacobian variety of $C^{\prime}$ over $k$. Take a closed point $s_{0}$ on $C^{\prime}$. Since the morphism $\left(C^{\prime}\right)^{g} \rightarrow J$ defined by $\left(s_{i}\right)_{i=1}^{g} \mapsto \sum_{i=1}^{g}\left(\left[s_{i}\right]-\left[s_{0}\right]\right)$ is surjective and the multiplication of $J$ by $n$ is surjective, there exists $\left(s_{i}\right)_{i=1}^{g} \in\left(C^{\prime}(k)\right)^{g}$ such that $u^{*}[s]-n\left[s_{0}\right]$ is linearly equivalent to $n \sum_{i=1}^{g}\left(\left[s_{i}\right]-\left[s_{0}\right]\right)$, which proves the lemma. 
Proposition 5.6. Let $(C, B)$ be a connected proper tame orbifold $k$-curve with \# Supp $B=1$. Assume that the genus of $C$ is positive. Then there exists an orbifold trivialization of $(C, B)$ that is the composite $u \circ v: C^{\prime \prime} \rightarrow C^{\prime} \rightarrow C$ of two finite coverings where $u$ is étale and $v$ is totally ramified over each branch point.

Proof. Take $s \in \operatorname{Supp} B$. Put $n:=\left[B_{S}: K_{S}\right]$. Take a connected étale covering space $u: C^{\prime} \rightarrow C$ and a rational function $h$ on $C^{\prime}$ given by Lemma 5.5. By $v: C^{\prime \prime} \rightarrow C^{\prime}$ we denote the normal model of the equation $z^{n}=h$. Then $u \circ v$ induces a desired orbifold trivialization.

Proposition 5.7. Let $(C, B)$ be a connected tame orbifold $k$-curve. Put $M:=$ \# Supp B. For each $s \in \operatorname{Supp} B$, we put $n_{s}:=\left[B_{s}, K_{s}\right]$. Then there exists an orbifold trivialization of $(C, B)$ if and only if neither of the following conditions are satisfied: (a) $C \cong \mathbb{P}_{k}^{1}$ and $M=1$; (b) $C \cong \mathbb{P}_{k}^{1}, M=2$, and $n_{s} \neq n_{t}$ where Supp $B=\{s, t\}$.

Proof. In the proof of the "if" part, we may replace $C$ by the smooth compactification of $C$. Thus, we may assume that $C$ is proper over $k$. By Proposition 3.18, we have only to consider the following cases: (1) $g(C)=0$ and $M \leq 2 ;(2) g(C)=0$, $M=3$, and $\operatorname{gcd}\left(n_{s}, n_{t}\right)=1$ for $s \neq t$; (3) $g(C)>0$ and $M=1$. Cases (1), (2), and (3) follow from Propositions 5.3, 5.4, and 5.6, respectively.

In the following, we provide steps in order to prove Proposition 5.12. Assume that $p>0$. Let $C$ be a connected proper smooth $k$-curve. We denote the sheaf of rational functions on $C$ by $\mu_{C}$. Put $\mathscr{P}_{C}:=\mu_{C} / \mathscr{O}_{C}$. The exact sequence of abelian sheaves $0 \rightarrow \mathrm{O}_{C} \rightarrow \mu_{C} \rightarrow \mathscr{P}_{C} \rightarrow 0$ induces a long exact sequence

$$
H^{0}\left(C, \mu_{C}\right) \stackrel{\phi_{C}}{\longrightarrow} H^{0}\left(C, \mathscr{P}_{C}\right) \stackrel{\psi_{C}}{\longrightarrow} H^{1}\left(C, \mathscr{O}_{C}\right) .
$$

By $F_{C}$ we denote the absolute Frobenius endomorphism of $C$ and its actions on the cohomology groups $H^{0}\left(C, \mathscr{P}_{C}\right)$ and $H^{1}\left(C, \mathscr{O}_{C}\right)$. We define $F_{C}^{0}$ as the identity map and, for each positive integer $d$, we inductively define $F_{C}^{d}$ by $F_{C}^{d}:=F_{C}^{d-1} \circ F_{C}$.

Lemma 5.8. For any $\xi \in H^{0}\left(C, \mathscr{P}_{C}\right)$, there exists a connected étale covering space $u: C^{\prime} \rightarrow C$, a nonnegative integer $d$, and a rational function $h$ on $C^{\prime}$ such that $u^{*} F_{C}^{d} \xi=\phi_{C^{\prime}}(h)$, where $u^{*}$ is the homomorphism $H^{0}\left(C, \mathscr{P}_{C}\right) \rightarrow H^{0}\left(C^{\prime}, \mathscr{P}_{C^{\prime}}\right)$ induced by $u$.

Proof. Since the $k$-vector space $H^{1}\left(C, O_{C}\right)$ is finite-dimensional, we may take a nonnegative integer $d$ and a polynomial $G(X)=\sum_{i=0}^{n} c_{i} X^{i} \in k[X]$ so that $c_{0} \neq 0$ and $G\left(F_{C}\right) \eta=0$, where we put $\eta:=F_{C}^{d} \psi_{C} \xi$. Take an affine covering $\mathcal{U}=\left\{U_{i}\right\}$ of $C$ and a representative $\left\{a_{i j}\right\} \in C^{1}\left(\mathcal{U}, \widehat{O}_{C}\right)$ of $\eta$. We define a polynomial $G^{(p)}(X) \in k[X]$ by $G^{(p)}(X):=\sum_{i=0}^{n} c_{i} X^{p^{i}}$. Since $G\left(F_{C}\right) \eta=0$, there exists 
$\left\{a_{i}\right\} \in C^{0}\left(\mathcal{U}, \mathscr{O}_{C}\right)$ such that $G^{(p)}\left(a_{i j}\right)=a_{j}-a_{i}$ for all $i$ and all $j$. Thus, we may define an étale covering space $u: C^{\prime} \rightarrow C$ by the equations

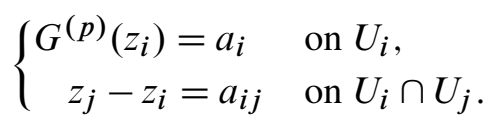

By definition, the pullback of $\eta$ via $u$ splits, which proves the lemma.

We denote the cokernel of an endomorphism $\phi$ of a module $M$ by $M_{\phi}$.

Lemma 5.9. Let $R$ be an excellent discrete valuation ring of positive characteristic with separably closed residue field. By $\widehat{R}$ we denote the completion of $R$ with respect to the maximal ideal. Put $K:=\operatorname{Frac} R, K^{\mathrm{sh}}:=\operatorname{Frac} R^{\mathrm{sh}}$, and $\hat{K}:=\operatorname{Frac} \hat{R}$. We denote the Frobenius endomorphisms on these fields by $F$. The canonical inclusion homomorphisms $K \rightarrow K^{\mathrm{sh}}$ and $K^{\mathrm{sh}} \rightarrow \hat{K}$ induce the canonical homomorphisms $\alpha: K \rightarrow K_{F-1}^{\mathrm{sh}}$ and $\beta: K_{F-1}^{\mathrm{sh}} \rightarrow \hat{K}_{F-1}$, respectively. Then:

(1) $\alpha$ is surjective and $R \subset \operatorname{Ker} \alpha$.

(2) $\beta$ is an isomorphism.

Proof. Since $K^{\text {sh }}$ is algebraically closed in $\widehat{K}$ by the approximation property [Bosch et al. 1990, 3.6.9], Artin-Schreier theory shows that $\beta$ is injective. Since $\hat{R}$ is isomorphic to the formal power series ring over the separably closed residue field of $R$, the relation $\widehat{R} \subset(F-1) \widehat{K}$ holds, which implies that $\beta \circ \alpha$ is surjective. Thus, Statement (2) holds, which implies Statement (1).

We recall the definition of the addition of the ring of Witt vectors $W(A)$ with coefficient ring $A$. Let $n$ be a nonnegative integer. Put

$$
W_{n}\left(X_{0}, \ldots, X_{n}\right):=\sum_{i=0}^{n} p^{i} X_{i}^{p^{n-i}} \in \mathbb{Z}\left[X_{0}, \ldots, X_{n}\right] .
$$

We inductively define $S_{n}$ as the unique polynomial in $\mathbb{Z}\left[X_{0}, \ldots, X_{n}, Y_{0}, \ldots, Y_{n}\right]$ satisfying the equality $W_{n}\left(S_{0}, \ldots, S_{n}\right)=W_{n}\left(X_{0}, \ldots, X_{n}\right)+W_{n}\left(Y_{0}, \ldots, Y_{n}\right)$. For $a=\left(a_{0}, \ldots, a_{n}, \ldots\right) \in W(A)$ and $b=\left(b_{0}, \ldots, b_{n}, \ldots\right) \in W(A)$, the addition of Witt vectors is defined by

$$
a+b:=\left(S_{0}\left(a_{0}, b_{0}\right), \ldots, S_{n}\left(a_{0}, \ldots, a_{n}, b_{0}, \ldots, b_{n}\right), \ldots\right) .
$$

Lemma 5.10. We denote the ideal of $\mathbb{Z}\left[X_{0}, \ldots, X_{n}, Y_{0}, \ldots, Y_{n}\right]$ generated by $\left\{X_{i} Y_{j}\right\}_{0 \leq i, j \leq n}$ by $I$. Then the equality $S_{n} \equiv X_{n}+Y_{n} \bmod I$ holds. In particular, the equality

$$
\left(a_{0}, \ldots, a_{n-1}, a_{n}, \ldots\right)+\left(0, \ldots, 0, b_{n}, \ldots\right)=\left(a_{0}, \ldots, a_{n-1}, a_{n}+b_{n}, \ldots\right)
$$

holds in $W(A)$. 
Proof. Let us show the first equality by induction on $n$. The case $n=0$ is clear. Assume that the case $i$ is proved for any $i<n$. By the induction hypothesis, the equality $p^{n} S_{n} \equiv p^{n} X_{n}+p^{n} Y_{n}$ mod $I$ holds, which proves the case $n$. Thus, the first equality holds for any $n$. The first equality shows the last equality.

For a positive integer $n$, we denote the ring of length- $n$ Witt vectors with coefficient ring $A$ by $W_{n}(A)$. We denote the Frobenius endomorphism on $W_{n}(A)$ by $F$. Take a connected étale covering space $u: C^{\prime} \rightarrow C$, a closed point $s$ on $C$, and $s^{\prime} \in u^{-1}(s)$. Put $K_{s}:=\operatorname{Frac} \mathcal{O}_{C, s}^{\text {sh }}$ and $K_{s^{\prime}}^{\prime}:=$ Frac $0_{C^{\prime}, s^{\prime}}^{\text {sh }}$. The extensions $K_{s^{\prime}}^{\prime} / K_{s}$ for all $s^{\prime} \in u^{-1}(s)$ induce a homomorphism

$$
\Delta_{u, s, n}: W_{n}\left(K_{s}\right)_{F-1} \rightarrow \bigoplus_{s^{\prime} \in u^{-1}(s)} W_{n}\left(K_{s^{\prime}}^{\prime}\right)_{F-1} .
$$

Put $A_{u, s}:=\mathbb{O}_{C^{\prime}}\left(C^{\prime}-u^{-1}(s)\right)$. The canonical homomorphisms $A_{u, s} \rightarrow K_{s^{\prime}}^{\prime}$ for all $s^{\prime} \in u^{-1}(s)$ induce a homomorphism

$$
\phi_{u, s, n}: W_{n}\left(A_{u, s}\right)_{F-1} \rightarrow \bigoplus_{s^{\prime} \in u^{-1}(s)} W_{n}\left(K_{s^{\prime}}^{\prime}\right)_{F-1} .
$$

Put $\Delta_{C, s, n}:=\lim _{\longrightarrow} \Delta_{u, s, n}$ and $\phi_{C, s, n}:=\lim _{C} \phi_{u, s, n}$, where $u: C^{\prime} \rightarrow C$ runs through all connected étale covering spaces of $C$. By construction, the homomorphisms $\Delta_{C, s, n}$ and $\phi_{C, s, n}$ are compatible with the reductions of the rings of Witt vectors.

Lemma 5.11. The relation $\operatorname{Im} \Delta_{C, s, n} \subset \operatorname{Im} \phi_{C, s, n}$ holds.

Proof. Take $\xi \in W_{n}\left(K_{s}\right)_{F-1}$. Put $\eta:=\Delta_{C, s, n}(\xi)$. We have to show that $\eta \in$ $\operatorname{Im} \phi_{C, s, n}$. By induction on $n$, we have only to consider the following cases: (1) $n=1$; (2) $n>1$ and $\xi$ is contained in the kernel of the reduction homomorphism $W_{n}\left(K_{S}\right) \rightarrow W_{n-1}\left(K_{S}\right)$. Note that $W_{1}(A)=A$ for any ring $A$. Lemma 5.10 reduces Case (2) to Case (1). Thus, we may assume that $n=1$. Lemma 5.9(1) shows that the canonical homomorphism $\mu_{C, s} \rightarrow K_{s}$ induces a surjective homomorphism $P: \mathscr{P}_{C, s} \rightarrow\left(K_{s}\right)_{F-1}$. Thus, we have only to show that $\Delta_{C, s, 1}(P(\theta)) \in \operatorname{Im} \phi_{C, s, 1}$ for any $\theta \in \mathscr{P}_{C, s}$. Since the equality $X^{d}=1+\left(\sum_{i=0}^{d-1} X^{i}\right)(X-1)$ holds in the polynomial ring $k[X]$ for any positive integer $d$, we may replace $\theta$ by $F^{d} \theta$ for any positive integer $d$. Thus, the lemma follows from Lemma 5.8.

Proposition 5.12. Assume that $p>0$. Let $(C, B)$ be a connected proper cyclic $p$-orbifold $k$-curve with \# Supp $B=1$. Then there exists an orbifold trivialization of $(C, B)$ that is the composite $u \circ v: C^{\prime \prime} \rightarrow C^{\prime} \rightarrow C$ of two finite coverings where $u$ is étale and $v$ is totally ramified over each branch point.

Proof. Take $s \in \operatorname{Supp} B$. Take an integer $n$ so that $\left[B_{s}: K_{s}\right]=p^{n}$. Since $H^{1}\left(K_{s}, W_{n}\right)=0$, the exact sequence of $\operatorname{Gal}\left(\overline{K_{S}} / K_{S}\right)$-modules

$$
0 \longrightarrow \mathbb{Z} / p^{n} \mathbb{Z} \longrightarrow W_{n}\left(\overline{K_{S}}\right) \stackrel{F-1}{\longrightarrow} W_{n}\left(\overline{K_{S}}\right) \longrightarrow 0
$$


induces an isomorphism $H^{1}\left(K_{S}, \mathbb{Z} / p^{n} \mathbb{Z}\right) \cong W_{n}\left(K_{S}\right)_{F-1}$. In particular, the field extension $B_{S} / K_{S}$ is induced by an element $\xi \in W_{n}\left(K_{S}\right)_{F-1}$. Lemma 5.11 gives a connected étale covering space $u: C^{\prime} \rightarrow C$ and an element $\eta \in W_{n}\left(A_{u, s}\right)_{F-1}$ such that the equality $\Delta_{u, s, n}(\xi)=\phi_{u, s, n}(\eta)$ holds. By $K^{\prime}$ we denote the function field of $C^{\prime}$. The image of $\eta$ under the canonical homomorphism $W_{n}\left(A_{u, s}\right)_{F-1} \rightarrow W_{n}\left(K^{\prime}\right)_{F-1}$ induces a cyclic extension $K^{\prime \prime} / K^{\prime}$ of degree $p^{n}$. Take the normalization $v: C^{\prime \prime} \rightarrow C^{\prime}$ of $C^{\prime}$ in $K^{\prime \prime}$. By the choice of $\eta$, the morphism $u \circ v$ induces a desired orbifold trivialization.

Proposition 5.13. Let $(C, B)$ be a connected $p$-orbifold $k$-curve. Then there exists an orbifold trivialization of $(C, B)$.

Proof. We may replace $C$ by the smooth compactification of $C$. Thus, we may assume that $C$ is proper over $k$. By Proposition 3.18, we may assume that \# Supp $B=1$. Take $s \in \operatorname{Supp} B$. Put $m:=\left[B_{s}: K_{s}\right]$. Let us show the proposition by induction on $m$. The case $m=1$ is clear. Assume that $m>1$. Since $B_{S} / K_{S}$ is solvable, we may take a Galois extension $B_{s}^{\prime} / K_{s}$ of degree $p$ in $B_{s}$. For each $t \in P(C) \backslash\{s\}$, we put $B_{t}^{\prime}:=K_{t}$. We define a map $B^{\prime}$ on $P(C)$ by $t \mapsto B_{t}^{\prime} / K_{t}$ (Definition 3.6). Then the pair $\left(C, B^{\prime}\right)$ is an orbifold. Applying Proposition 5.12 to $\left(C, B^{\prime}\right)$, we may reduce the case $m$ to the case $m / p$. Thus, the case $m$ holds by the induction hypothesis.

Definition 5.14. The Euler characteristic $e(C)$ of a proper smooth $k$-curve $C$ is the $\ell$-adic Euler characteristic of $C$, which does not depend on the choice of the prime number $\ell$ that is prime to $p$. Let $(C, B)$ be a proper orbifold $k$-curve. Take $s \in \operatorname{Supp} B$. We use the notation $B_{s} / K_{s}$ introduced in Definition 3.6. By $B_{s}^{\circ}$ and $K_{S}^{\circ}$ we denote the valuation rings of the discrete valuation fields $B_{S}$ and $K_{s}$, respectively. We define the orbifold Euler characteristic $e(C, B)$ of $(C, B)$ by

$$
e(C, B):=e(C)-\sum_{s \in \operatorname{Supp} B} \frac{1}{\left[B_{S}: K_{S}\right]} \text { length }_{K_{s}^{\circ}}\left(\Omega_{B_{S}^{\circ} / K_{s}^{\circ}}^{1}\right) .
$$

The Riemann-Hurwitz formula shows the following:

Proposition 5.15. Let $(C, B)$ and $\left(C^{\prime}, B^{\prime}\right)$ be two proper orbifold $k$-curves. If there exists an orbifold étale morphism $\left(C^{\prime}, B^{\prime}\right) \rightarrow(C, B)$ of degree $n$, then the equality $e\left(C^{\prime}, B^{\prime}\right)=n e(C, B)$ holds. In particular, if $e(C, B)>0$ and $C$ is connected, then the underlying curve of any connected orbifold étale covering space of $(C, B)$ is isomorphic to $\mathbb{P}_{k}^{1}$.

Proof of Theorem 1.3. Let us show Statement (2). Assume that $C$ is not proper over $k$. Take the smooth compactification $\bar{C}$ of $C$. We may choose an extension $\bar{B}$ of $B$ to $P(\bar{C})$ so that the orbifold $(\bar{C}, \bar{B})$ satisfies neither Condition (a) nor Condition (b). Thus, we may assume that $C$ is proper over $k$. First, we consider 
the "if" part. By Propositions 3.18, 5.15, and 5.13, we may assume that $B^{t}=B$. In that case, the "if" part follows from Proposition 5.7. Next, we consider the "only if" part. Take $s \in \operatorname{Supp} B$ and an orbifold trivialization $C_{1} \rightarrow(C, B)$. By Proposition 5.7, we have only to show that Condition (a) is not satisfied. Assume that Condition (a) is satisfied. Since $C$ is simply connected, Proposition 5.12 gives an orbifold trivialization $u: C_{2} \rightarrow\left(C, B^{w}\right)$ that is totally ramified over the unique branch point $s$. Condition (a) and Proposition 5.15 imply that $C_{2} \cong \mathbb{P}_{k}^{1}$. Take the normalization $C_{3}$ of $C_{1} \times{ }_{C} C_{2}$ and the canonical projection $v: C_{3} \rightarrow C_{2}$. Lemma 3.14 shows that $v$ is tamely ramified over the unique branch point $u^{-1}(s)$, which contradicts Lemma 5.2. Thus, Condition (a) is not satisfied.

Let us show Statement (1). The "if part" follows from Proposition 5.3. We consider the "only if" part. Assume that Condition (a) is not satisfied. Statement (2) implies that $C \cong \mathbb{P}_{k}^{1}$ and $M \leq 2$. Proposition 5.13 implies that $B^{t}=B$. Proposition 5.3 implies that $\operatorname{gcd}\left(n_{s}, n_{t}\right)=1$ for $s \neq t$. Thus, Condition (b) is satisfied. Therefore, the "only if" part holds.

\section{Fundamental groups of elliptic fibrations}

6A. Elliptic fibrations. We study elliptic surfaces by localizing the fibrations with respect to the base curves. To this end, we generalize the definition of elliptic surfaces. We refer to [Liu 2002, §§8-9] for fibered surfaces.

Definition 6.1. An elliptic fibration is a triple $(X, C, f)$ satisfying the following conditions:

(1) $C$ and $X$ are excellent regular integral schemes of dimension one and two, respectively.

(2) $f: X \rightarrow C$ is a proper morphism.

(3) The homomorphism $\mathrm{O}_{C} \rightarrow f_{*} \mathrm{O}_{X}$ associated to $f$ is an isomorphism.

(4) The generic fiber of $f$ is a proper smooth curve of genus one.

Let $(X, C, f)$ be an elliptic fibration. A prime divisor $D$ on $X$ is said to be a $(-1)$-curve if the following conditions are satisfied. Put $k:=\Gamma\left(D, O_{D}\right)$. Then $D$ is $k$-isomorphic to $\mathbb{P}_{k}^{1}$ and $\left.\operatorname{deg} O_{X}(D)\right|_{D}=-1$. If any fiber of $f$ does not contain a (-1)-curve, then $(X, C, f)$ is said to be relatively minimal. The multiplicity of a closed fiber $F$ of $f$ is the multiplicity of the divisor $F$ on $X$ (Section 2). The minimal regular $C$-model of the Jacobian of the generic fiber of $f$ is called the Jacobian fibration of $f$.

Remark 6.2. Conditions (2) and (3) show that $f$ is surjective. Thus, Condition (1) shows that $f$ is flat. The multiplicity of $F$ does not depend on the choice of the proper regular $C$-model of the generic fiber of $f$ [Liu 2002, 9.2.7]. 
Lemma 6.3. Let $(X, C, f)$ be an elliptic fibration and $\xi: Y \rightarrow X$ a connected étale covering space. Take the Stein factorization

$$
Y \stackrel{h}{\longrightarrow} D \stackrel{v}{\longrightarrow} C
$$

of the composite $f \circ \xi: Y \rightarrow X \rightarrow C$. Then:

(1) $(Y, D, h)$ is an elliptic fibration.

(2) $v$ is finite, flat, surjective, and generically separable.

Choose an integral scheme $C^{\prime}$ and a finite flat morphism $u: C^{\prime} \rightarrow C$ such that $v$ factors through $u$. Take the normalization $X^{\prime}$ of $X \times_{C} C^{\prime}$, the canonical projections $u^{\prime}: X^{\prime} \rightarrow X$ and $f^{\prime}: X^{\prime} \rightarrow C^{\prime}$, and the unique morphism $\xi^{\prime}: Y \rightarrow X^{\prime}$ satisfying $\xi=u^{\prime} \circ \xi^{\prime}$ and $h=f^{\prime} \circ \xi^{\prime}$. Then:

(3) $\left(X^{\prime}, C^{\prime}, f^{\prime}\right)$ is an elliptic fibration.

(4) $u^{\prime}$ and $\xi^{\prime}$ are finite, étale, and surjective.

Proof. Since $X$ is regular and $\xi$ is étale, the scheme $Y$ is regular. Thus, Statements (1) and (2) follow from Proposition 4.5. Statement (4) follows from Lemma 4.7. Since $X$ is regular and $u^{\prime}$ is étale, the scheme $X^{\prime}$ is regular. Thus, Statement (3) follows from Proposition 4.5.

We frequently use the following:

Proposition 6.4 [Liu et al. 2004, 6.6]. Let C be the spectrum of a complete discrete valuation ring with algebraically closed residue field and field of fractions $K$. Let $(X, C, f)$ be a relatively minimal elliptic fibration with Jacobian fibration $(E, C, g)$. By $X_{K}$ and $E_{K}$ we denote the generic fibers of $f$ and $g$, respectively. Then the special fiber of $f$ is of type ${ }_{m} T$ (the Kodaira symbol) if and only if the special fiber of $g$ is of type $T$ and the order of the torsor $\left[X_{K}\right] \in H^{1}\left(K, E_{K}\right)$ is equal to $m$.

We refer to [Liu 2002, 8.3.39, 8.3.44, 9.3.31, and 9.3.32] for desingularizations and the minimal desingularizations of fibered surfaces.

Lemma 6.5. Let $(X, C, f)$ be an elliptic fibration with generic fiber $X_{K}$ and $\xi_{K}: Y_{K} \rightarrow X_{K}$ a finite morphism between geometrically connected $K$-curves of genus one. Take the normalization $\xi: Y \rightarrow X$ of $X$ in $\xi_{K}$. Assume that $f$ is smooth and that the residue field at any closed point on $C$ is algebraically closed. Then the triple $(Y, C, f \circ \xi)$ is a relatively minimal elliptic fibration.

Proof. We may assume that $C$ is the spectrum of a discrete valuation ring. Take the minimal desingularization $\lambda: \hat{Y} \rightarrow Y$ of $Y$. By $X_{k}, Y_{k}$, and $\hat{Y}_{k}$ we denote the special fibers of $f, f \circ \xi$, and $f \circ \xi \circ \lambda$, respectively. Choose an irreducible component $D$ of $Y_{k}$ and an irreducible component $\widehat{D}$ of $\widehat{Y}_{k}$ dominating $D$. Since $1=g\left(X_{k}\right) \leq g(D) \leq g(\hat{D})$, the Néron-Kodaira classification of singular fibers 
implies that $\widehat{D}$ is the unique irreducible component of $\hat{Y}_{k}$ whose geometric genus is equal to 1 . Thus, the component $D$ is the unique irreducible component of $Y_{k}$, and the component $\hat{D}$ is the unique irreducible component of $\widehat{Y}_{k}$ that dominates $D$. Since $\lambda$ is the minimal desingularization, the morphism $\lambda$ is an isomorphism. Therefore, the triple $(Y, C, f \circ \xi)$ is a relatively minimal elliptic fibration.

Lemma 6.6. Let $(X, C, f)$ be an elliptic fibration with Jacobian fibration $(E, C, g)$. Assume that the reduction of any closed fiber of $f$ is isomorphic to an elliptic curve and that the residue field at any closed point on $C$ is algebraically closed. By $X_{K}$ and $E_{K}$ we denote the generic fibers of $f$ and $g$, respectively. Take a positive multiple $n$ of the order of the torsor $\left[X_{K}\right] \in H^{1}\left(K, E_{K}\right)$. Then there exists a $C$-morphism $X \rightarrow E$ whose restriction to the generic fibers induces the multiplication of their Jacobian $E_{K}$ by $n$.

Proof. Take a finite Galois extension $K^{\prime} / K$ so that $X\left(K^{\prime}\right) \neq \varnothing$. Put $G:=$ $\operatorname{Gal}\left(K^{\prime} / K\right)$ and $X_{K^{\prime}}:=X_{K} \times_{K} K^{\prime}$. Choose a cocycle $c \in Z^{1}\left(G, E\left(K^{\prime}\right)\right)$ representing $\left[X_{K}\right]$. The curve $X_{K} / K$ may be obtained as the quotient of a $G$-equivariant action on $X_{K^{\prime}} / K^{\prime}$ induced by $c$ (see Section 6B). Moreover, an endomorphism of $Z^{1}\left(G, E\left(K^{\prime}\right)\right)$ induces a $G$-equivariant endomorphism of $X_{K^{\prime}}$, whose quotient is a $K$-morphism between torsors of $E_{K}$. Since the endomorphism on $H^{1}\left(K, E_{K}\right)$ induced by the multiplication of $E_{K}$ by $n$ maps the torsor $\left[X_{K}\right]$ to the trivial torsor $\left[E_{K}\right]$, the endomorphism induces a $K$-morphism $\xi_{K}: X_{K} \rightarrow E_{K}$. Take the normalization $Y \rightarrow E$ of $E$ in $\xi_{K}$. Proposition 6.4 shows that $g$ is smooth. By Lemma 6.5, the scheme $Y$ is the minimal regular $C$-model of $X_{K}$. Since the minimal regular $C$-model of $X_{K}$ is unique up to unique $C$-isomorphism, the $C$ scheme $Y$ is $C$-isomorphic to $X$, which concludes the proof.

Corollary 6.7. Let $C$ be the spectrum of a complete discrete valuation ring with algebraically closed residue field. Let $(X, C, f)$ be a relatively minimal elliptic fibration with Jacobian fibration $(E, C, g)$. Then the reduction of the special fiber of $f$ is isomorphic to an ordinary elliptic curve if and only if the special fiber of $g$ is an ordinary elliptic curve.

6B. Étale coverings of local elliptic fibrations. Let $R$ be a complete discrete valuation ring with algebraically closed residue field $k$ of characteristic $p \geq 0$ and field of fractions $K$. Put $C:=\operatorname{Spec} R$. Let $(X, C, f)$ be an elliptic fibration with Jacobian fibration $(E, C, g)$. By $X_{K}$ and $E_{K}$ we denote the generic fibers of $f$ and $g$, respectively. By $\left[X_{K}\right]$ we denote the element of $H^{1}\left(K, E_{K}\right)$ corresponding to the torsor $X_{K}$ of $E_{K}$. Take a separable closure $\bar{K}$ of $K$. Take the maximal base field $\widetilde{K}$ of $f$ in $\bar{K}$ (Definition 4.9). In this subsection, we determine the extension $\widetilde{K} / K$.

Take a finite Galois extension $K^{\prime} / K$ in $\bar{K}$ so that $X\left(K^{\prime}\right) \neq \varnothing$. Put $G_{K^{\prime} / K}:=$ $\operatorname{Gal}\left(K^{\prime} / K\right)$. The group $H^{1}\left(G_{K^{\prime} / K}, E\left(K^{\prime}\right)\right)$ may be regarded as a subgroup of 
$H^{1}\left(K, E_{K}\right)$ by the inflation homomorphism. Then the torsor $\left[X_{K}\right] \in H^{1}\left(K, E_{K}\right)$ is contained in $H^{1}\left(G_{K^{\prime} / K}, E\left(K^{\prime}\right)\right)$ since $\left[X_{K}\right]$ splits over $K^{\prime}$. Choose a cocycle $c \in Z^{1}\left(G_{K^{\prime} / K}, E\left(K^{\prime}\right)\right)$ representing $\left[X_{K}\right]$. The extension $K^{\prime} / K$ induces a finite covering $C^{\prime} / C$. By $\left(E^{\prime}, C^{\prime}, g^{\prime}\right)$ we denote the Jacobian fibration of $X_{K} \times{ }_{K} K^{\prime}$. By the uniqueness of the normalization $C^{\prime}$ of $C$ in $K^{\prime}$ and the Jacobian fibration $g^{\prime}$, we obtain a homomorphism $\rho: G_{K^{\prime} / K} \rightarrow \operatorname{Aut}\left(C^{\prime} / C\right) \rightarrow \operatorname{Aut}\left(E^{\prime} / C\right)$, where the first arrow is induced by the automorphisms on the generic point of $C^{\prime}$ and the second arrow is induced by the base change of the automorphisms via $g^{\prime}$. Furthermore, we obtain a map $\tau: G_{K^{\prime} / K} \rightarrow E\left(K^{\prime}\right) \rightarrow \operatorname{Aut}\left(E^{\prime} / C^{\prime}\right)$, where the first arrow is given by $c$ and the second arrow is induced by the translation by addition. Since $c$ is a cocycle, the map $\tilde{\tau}: G_{K^{\prime} / K} \rightarrow \operatorname{Aut}\left(E^{\prime} / C\right)$ defined by $\sigma \mapsto \tau(\sigma) \circ \rho(\sigma)$ is a homomorphism. By $\chi: E^{\prime} \rightarrow Z:=E^{\prime} / \operatorname{Im} \tilde{\tau}$ we denote the quotient morphism of the action $\tilde{\tau}$. The quotient $Z$ is a normal scheme over $C$ whose generic fiber is isomorphic to $X_{K}$.

Lemma 6.8. If any element of $\operatorname{Im} \tilde{\tau}$ fixes any closed point on $E^{\prime}$, then $\tilde{K}=K$.

Proof. We may assume that $X$ is the minimal desingularization of $Z$. Since $Z$ is normal, we may take a regular closed point $z$ on $Z$. By $x \in X$ we denote the preimage of $z$. The extension $\tilde{K} / K$ induces a finite covering $\widetilde{C} / C$. Take the minimal desingularization $X^{\prime}$ of $X \times_{C} C^{\prime}$ (resp. $\tilde{X}$ of $X \times_{C} \tilde{C}$ ) and the canonical projection $u^{\prime}: X^{\prime} \rightarrow X$ (resp. $\tilde{u}: \tilde{X} \rightarrow X$ ). By the choice of $x$, the preimage $\left(u^{\prime}\right)^{-1}(x)$ consists of one closed point on $X^{\prime}$. By Proposition 4.15(4) and the definitions of $u^{\prime}$ and $\tilde{u}$, the morphism $u^{\prime}$ factors through the finite étale surjective morphism $\tilde{u}$. Thus, the degree of $\tilde{u}$ is equal to one, which implies that $\tilde{K}=K$.

Assume that $(X, C, f)$ is relatively minimal. Put $G_{K}:=\operatorname{Gal}(\bar{K} / K)$. By ${ }_{m} T$ we denote the type of the special fiber of $f$ (the Kodaira symbol). The type $T$ is divided into the following three cases, (A), (M), and (E):

Case (A). Additive type: $T \neq \mathrm{I}_{n}(n \geq 0)$. Since the residue field $k$ of $R$ is algebraically closed and the special fiber of $f$ is simply connected, Lemma 6.3 implies that $X$ is simply connected. In particular, the equality $\widetilde{K}=K$ holds.

Case $(M)$. Multiplicative type: $T=\mathrm{I}_{n}(n>0)$. Tate's uniformization gives an exact sequence of $G_{K}$-modules

$$
0 \longrightarrow \mathbb{Z} \longrightarrow \mathbb{G}_{m, K}(\bar{K}) \stackrel{\pi}{\longrightarrow} E_{K}(\bar{K}) \longrightarrow 0,
$$

where $\pi$ maps 1 to $q \in K$ satisfying $0<|q|<1$. The exact sequence induces a long exact sequence

$$
H^{1}\left(K, \mathbb{G}_{m, K}\right) \longrightarrow H^{1}\left(K, E_{K}\right) \longrightarrow H^{2}(K, \mathbb{Z}) \longrightarrow H^{2}\left(K, \mathbb{G}_{m, K}\right) .
$$


Since $H^{1}\left(K, \mathbb{G}_{m, K}\right)=H^{2}\left(K, \mathbb{G}_{m, K}\right)=0$ and $H^{2}(K, \mathbb{Z}) \cong \operatorname{Hom}\left(G_{K}, \mathbb{Q} / \mathbb{Z}\right)$, we obtain an isomorphism $\phi_{M}: H^{1}\left(K, E_{K}\right) \cong \operatorname{Hom}\left(G_{K}, \mathbb{Q} / \mathbb{Z}\right)$. Put $\gamma_{M}:=\phi_{M}\left(\left[X_{K}\right]\right)$. The group $\operatorname{Im} \gamma_{M}$ is finite and cyclic. The Galois extension $L / K$ corresponding to $\operatorname{Ker} \gamma_{M}$ is the minimum separable field extension that splits $\left[X_{K}\right]$. By $D$ we denote the normalization of $C$ in $L$. Put $d:=[L: K]$. Then the normalization $Y$ of $X \times_{C} D$ is a relatively minimal elliptic fibration over $D$ with special fiber of type $\mathrm{I}_{d n}$, and the induced morphism $Y \rightarrow X$ is étale (see the proof of [Liu et al. 2004, 8.3(b)]). In particular, the relation $\widetilde{K} \supset L$ holds. Proposition 4.15(4) gives the relation $\widetilde{K} \subset L$. Thus, the equality $\widetilde{K}=L$ holds.

Case (E). Elliptic type: $T=\mathrm{I}_{0}$. By $\widehat{E}$ we denote the formal group law associated to $E$. By $\bar{R}$ and $\overline{\mathfrak{m}}$ we denote the integral closure of $R$ in $\bar{K}$ and the maximal ideal of $\bar{R}$, respectively. Then $\widehat{E}$ gives a group structure on $\overline{\mathfrak{m}}$. By $\widehat{E}(\overline{\mathfrak{m}})$ we denote this group. Since the canonical homomorphism $E(\bar{R}) \rightarrow E(\bar{K})$ is a $G_{K}$-module isomorphism by the valuative criterion for properness, we obtain an exact sequence of $G_{K}$-modules

$$
0 \longrightarrow \widehat{E}(\overline{\mathfrak{m}}) \longrightarrow E(\bar{K}) \longrightarrow E(k) \longrightarrow 0 .
$$

The exact sequence induces a long exact sequence

$$
0 \longrightarrow H^{1}(K, \hat{E}(\overline{\mathfrak{m}})) \stackrel{\iota}{\longrightarrow} H^{1}\left(K, E_{K}\right) \longrightarrow H^{1}(K, E(k)) \stackrel{\psi}{\longrightarrow} H^{2}(K, \widehat{E}(\overline{\mathfrak{m}})) .
$$

Since $G_{K}$ acts trivially on $E(k)$, we obtain an isomorphism $H^{1}(K, E(k)) \cong$ $\operatorname{Hom}\left(G_{K}, E(k)\right)$.

Lemma 6.9. If $p=0$, then the group $H^{i}(K, \widehat{E}(\overline{\mathfrak{m}}))$ is trivial for any positive integer $i$. Otherwise the group $H^{i}(K, \widehat{E}(\overline{\mathfrak{m}}))$ is p-primary for any positive integer $i$.

Proof. Any $i$-th Galois cohomology group is torsion for any positive integer $i$. Take an integer $n$ so that $p \nmid n$. Then the multiplication of $\widehat{E}(\overline{\mathfrak{m}})$ by $n$ is an isomorphism [Silverman 2009, IV.2.3(b)]. These facts show the lemma.

Lemma 6.10. The homomorphism $\psi$ is the zero map.

Proof. By Lemma 6.9, we may assume that $p>0$. Furthermore, we have only to show the image of any element of $H^{1}(K, E(k))$ of $p$-power order under $\psi$ is equal to zero. If the special fiber of $E$ is ordinary, then the statement follows from [Raynaud 1970, 9.4.1(iii)]. Otherwise, the group $E(k)$ is $p$-torsion free. Thus, the group $H^{1}(K, E(k))$ is $p$-torsion free, which implies that the statement holds.

From Lemma 6.10 we get a surjective homomorphism $\phi_{E}: H^{1}\left(K, E_{K}\right) \rightarrow$ $\operatorname{Hom}\left(G_{K}, E(k)\right)$. Put $\gamma_{E}:=\phi_{E}\left(\left[X_{K}\right]\right)$. The group $\operatorname{Im} \gamma_{E}$ is finite and cyclic. The Galois extension $L / K$ corresponding to $\operatorname{Ker} \gamma_{E}$ is the minimum separable field extension that splits the image of $\left[X_{K}\right]$ in $H^{1}(K, E(k))$. By $D$ we denote the normalization of $C$ in $L$. Put $d:=[L: K]$. Then the normalization $Y$ of $X \times_{C} D$ is 
a relatively minimal elliptic fibration over $D$ with special fiber of type ${ }_{m / d} \mathrm{I}_{0}$, and the canonical projection $Y \rightarrow X$ is étale. In particular, the relation $\widetilde{K} \supset L$ holds. If $\left[X_{K}\right] \in \iota\left(H^{1}\left(G_{K}, \widehat{E}(\overline{\mathfrak{m}})\right)\right)$, then any element of $\operatorname{Im} \tilde{\tau}$ fixes any closed point on $E^{\prime}$. Thus, Lemma 6.8 gives the equality $\widetilde{K}=L$.

We summarize the above results:

Proposition 6.11. Assume that $(X, C, f)$ is relatively minimal. By ${ }_{m} T$ we denote the type of the special fiber of $f$. Take the maximal base field $\widetilde{K}$ of $f$ (Definition 4.9). Then $[\tilde{K}: K]$ divides $m$ (Proposition 4.15(3)). Moreover:

(A) If $T \neq \mathrm{I}_{n}(n \geq 0)$, then $X$ is simply connected and $\widetilde{K}=K$.

(M) If $T=\mathrm{I}_{n}(n>0)$, then $\tilde{K} / K$ corresponds to $\operatorname{Ker} \gamma_{M}$ in Case $(M)$ and $[\tilde{K}: K]=$ $\# \operatorname{Im} \gamma_{M}=m$.

(E) If $T=\mathrm{I}_{0}$, then $\tilde{K} / K$ corresponds to $\operatorname{Ker} \gamma_{E}$ in Case $(E),[\tilde{K}: K]=\# \operatorname{Im} \gamma_{E}$, and one of the following statements holds: (1) $p=0$ and $[\widetilde{K}: K]=m ;(2) p>0$ and $m /[\widetilde{K}: K]$ is a power of $p$.

In particular, the extension $\tilde{K} / K$ is finite and cyclic.

Lemma 6.12. Assume that $(X, C, f)$ is relatively minimal. By $m$ we denote the multiplicity of the special fiber $X_{k}$ of $f$. We define a divisor $F$ on $X$ by $F:=X_{k} / m$. $B y n$ we denote the order of the normal bundle of $F$ in the Picard group Pic $F$. Then:

(1) The $\mathrm{O}_{C}$-module $R^{1} f_{*} \mathrm{O}_{X}$ is torsion-free if and only if the equality $m=n$ holds.

(2) If $p=0$, then the equality $m=n$ holds. Otherwise, there exists a nonnegative integer e such that the equality $m=n p^{e}$ holds.

(3) We use the notation introduced in Lemma 6.3. Take $m^{\prime}$ and $n^{\prime}$ for $\left(X^{\prime}, C^{\prime}, f^{\prime}\right)$ in the same way. By $d$ we denote the degree of $u$. Assume that $F$ is isomorphic to an elliptic curve and $p \nmid d$. Then the equalities $m=d m^{\prime}$ and $n=d n^{\prime}$ hold.

Proof. Statements (1) and (2) follow from Proposition 1 in [Mitsui 2013]. Let us show Statement (3). Since $u^{\prime}$ is étale, the equality $u \circ f^{\prime}=f \circ u^{\prime}$ gives the equality $m=d m^{\prime}$. By $\lambda: F^{\prime} \rightarrow F$ we denote the base change of $u^{\prime}$ via the inclusion morphism $F \rightarrow X$. Since $u^{\prime}$ is a finite étale surjective morphism of degree $d$, the base change $\lambda$ is a finite étale surjective morphism of degree $d$. Since $F$ is isomorphic to an elliptic curve, the morphism $\lambda$ may be regarded as a morphism between elliptic curves over $k$. The morphism $\lambda$ induces a homomorphism $\lambda^{*}: \operatorname{Pic} F \rightarrow \operatorname{Pic} F^{\prime}$. Since $u^{\prime}$ is étale, the divisor $F^{\prime}$ is equal to the pullback of the divisor $F$ via $u^{\prime}$, which implies that $N_{F^{\prime} / X}=\lambda^{*} N_{F / X}$. Since $p \nmid d$, the relation $p \nmid\left(n / n^{\prime}\right)$ holds. Thus, the equality $m=d m^{\prime}$ and Statement (2) give the equality $n=d n^{\prime}$.

Lemma 6.13 [EGA III 1 1961, 7.7.5(II), 7.8.4, and 7.9.4]. The following conditions are equivalent: 
(1) $f$ is cohomologically flat in dimension zero [EGA $\left.\mathrm{III}_{2} 1963,7.8 .1\right]$; i.e., the formation of the direct image $f_{*} \mathrm{O}_{X}$ commutes with any base change.

(2) $R^{1} f_{*} \mathrm{O}_{X}$ is torsion-free.

Lemma 6.14. We use the notation introduced in Lemma 6.3. Suppose that the reduction of the special fiber of $f$ is isomorphic to an elliptic curve and that $p \nmid \operatorname{deg} v$. Then $R^{1} f_{*} \mathrm{O}_{X}$ is torsion-free if and only if $R^{1} h_{*} \mathrm{O}_{Y}$ is torsion-free.

Proof. By [Raynaud 1970, 9.4.2] and Lemma 6.13, we may assume that $\xi^{\prime}$ is an isomorphism. Then the lemma follows from (1) and (3) of Lemma 6.12.

Proposition 6.15. Suppose that $p>0$. Assume that $(X, C, f)$ is relatively minimal. $B y_{m} T$ we denote the type of the special fiber of $f$. Take the maximal base field $\tilde{K}$ of $f$ (Definition 4.9). By $\left(X_{k}\right)_{\text {red }}$ we denote the reduction of the special fiber of $f$. Then $p \nmid[\tilde{K}: K]$ if and only if one of the following conditions is satisfied:

(1) $T \neq \mathrm{I}_{n}(n \geq 0)$.

(2) $p \nmid m$.

(3) $\left(X_{k}\right)_{\text {red }}$ is isomorphic to a supersingular elliptic curve.

(4) $\left(X_{k}\right)_{\text {red }}$ is isomorphic to an ordinary elliptic curve and $R^{1} f_{*} \mathrm{O}_{X}$ is torsion-free.

Proof. By (A) and (M) of Proposition 6.11, we have only to consider the case $T=\mathrm{I}_{0}$. By Proposition 6.11(E), the extension $\widetilde{K} / K$ corresponds to Ker $\gamma_{E}$ and the equality $[\tilde{K}: K]=\# \operatorname{Im} \gamma_{E}$ holds. If $\left(X_{k}\right)_{\text {red }}$ is isomorphic to a supersingular elliptic curve, then the group $\operatorname{Hom}\left(G_{K}, E(k)\right)$ is $p$-torsion free (Corollary 6.7). Thus, we may assume that $\left(X_{k}\right)_{\text {red }}$ is isomorphic to an ordinary elliptic curve. By Lemmas 6.3 and 6.14 , we may assume that $[\tilde{K}: K]$ is a power of $p$. Then the proposition follows from [Raynaud 1970, 9.4.1(iii)] and Lemma 6.13.

Proposition 6.16. Let $L / K$ be a finite cyclic extension in $\bar{K}$ and $\left(E^{\prime}, C, g^{\prime}\right)$ a relatively minimal elliptic fibration with section. By $E_{k}^{\prime}$ we denote the special fiber of $g^{\prime}$. Then the following two conditions are equivalent:

(1) There exists a relatively minimal elliptic fibration $\left(X^{\prime}, C, f^{\prime}\right)$ satisfying the following conditions:

(a) The maximal base field of $f^{\prime}$ is equal to $L$ (Definition 4.9).

(b) The Jacobian fibration of $f^{\prime}$ is given by $g^{\prime}$.

(2) The following conditions are satisfied:

(a) If $E_{k}^{\prime}$ is not of type $\mathrm{I}_{n}(n \geq 0)$, then $L=K$.

(b) If $p>0$ and $E_{k}^{\prime}$ is isomorphic to a supersingular elliptic curve, then $p \nmid[L: K]$. 
Proof. Propositions 6.11 and 6.15 show that Condition (1) implies Condition (2). Let us show the converse. We may assume that $E_{k}^{\prime}$ is of type $\mathrm{I}_{n}(n \geq 0)$. Put $d:=[L: K]$ and $G_{L / K}:=\operatorname{Gal}(L / K)$. First, we consider the case $n>0$. By assumption, there exists an element $\gamma_{M} \in \operatorname{Hom}\left(G_{L / K}, \mathbb{Q} / \mathbb{Z}\right) \subset \operatorname{Hom}\left(G_{K}, \mathbb{Q} / \mathbb{Z}\right)$ of order $d$. Since $\phi_{M}$ is surjective, the case $n>0$ follows from Proposition 6.11(M). Next, we consider the case $n=0$. By assumption, there exists an element $\gamma_{E} \in$ $\operatorname{Hom}\left(G_{L / K}, E(k)\right) \subset \operatorname{Hom}\left(G_{K}, E(k)\right)$ of order $d$. Since $\phi_{E}$ is surjective, the case $n=0$ follows from Proposition 6.11(E).

Proposition 6.17. Let $(Y, C, h)$ be a relatively minimal elliptic fibration with special fiber of type $\mathrm{I}_{n}(n \geq 0)$. Let $\xi: Y \rightarrow X$ be a finite étale surjective $C$-morphism of degree $d$. We regard the restriction $\xi_{K}: Y_{K} \rightarrow X_{K}$ of $\xi$ to the generic fibers as a homomorphism between elliptic curves, which is determined by the choice of an element of $Y(K)$. By $G$ we denote the subgroup of $Y(K)$ consisting of all d-torsion elements. Put $H:=\xi_{K}(G) . B y \bar{G}$ and $\bar{H}$ we denote the sets of the closures of all elements of $G$ and $H$ in $Y$ and $X$, respectively. Assume that $p \nmid d$. Then:

(1) $d \mid n, \# G=d^{2}$, and $\# H=d$.

(2) All elements of $\bar{G}$ are disjoint.

(3) All elements of $\bar{H}$ are disjoint.

(4) There exists an irreducible component of the special fiber of $f$ that intersects with all elements of $\bar{H}$, and any other irreducible component of the special fiber of $f$ is disjoint from all elements of $\bar{H}$.

Proof. Since $\xi$ is étale, the relation $d \mid n$ holds and the special fiber of $f$ is of type $\mathrm{I}_{l}$, where we set $l:=n / d$. We may regard the smooth loci $\hat{X}$ and $\hat{Y}$ of $f$ and $h$ as the Néron models of $X_{K}$ and $Y_{K}$, respectively. By the Néron mapping property, the homomorphism $\xi_{K}$ induces the unique $C$-homomorphism $\hat{\xi}: \hat{Y} \rightarrow \hat{X}$, which is the restriction of $\xi$ to the smooth loci $\hat{X}$ and $\hat{Y}$. The restriction $\hat{\xi}_{k}$ of $\hat{\xi}$ to the special fibers of $\hat{X}$ and $\hat{Y}$ is a finite étale surjective $k$-homomorphism of degree $d$. If $n=0$, then $\hat{\xi}_{k}$ is a homomorphism between elliptic curves. If $n>0$, then $\widehat{\xi}_{k}$ is the homomorphism

$$
\mathbb{G}_{m, k} \times(\mathbb{Z} / n \mathbb{Z}) \longrightarrow \mathbb{G}_{m, k} \times(\mathbb{Z} / l \mathbb{Z}), \quad(z, e \bmod n) \longmapsto(z, e \bmod l) .
$$

Thus, the closure of $G$ in $Y$ is finite and étale over $C$ [Bosch et al. 1990, 7.3.2], which concludes the proof.

6C. Elliptic surfaces with prescribed orbifolds. In this subsection, we use the following notation. Let $k$ be an algebraically closed field of characteristic $p \geq 0$ and $C$ a connected proper smooth $k$-curve with function field $K$. An elliptic fibration $(X, C, f)$ is said to be trivial if there exists an elliptic curve $F$ over $k$ such that $X$ is $C$-isomorphic to the $C$-scheme $F \times{ }_{k} C$. Recall the following result on the global-to-local map: 
Proposition 6.18 [Cossec and Dolgachev 1989, 5.4.6]. Let $E_{K}$ be an elliptic curve over $K$. Take the minimal regular $C$-model $(E, C, g)$ of $E_{K}$. Assume that the elliptic fibration $(E, C, g)$ is nontrivial. For each closed point $s$ on $C$, we put $K_{s}:=$ Frac $O_{C, s}^{\text {sh }}$ and $E_{K_{s}}:=E_{K} \times_{K} K_{s}$. Then the global-to-local map $H^{1}\left(K, E_{K}\right) \rightarrow \bigoplus_{s \in C} H^{1}\left(K_{s}, E_{K_{s}}\right)$ is surjective.

Theorem 6.19. Let $(C, B)$ be a connected cyclic orbifold $k$-curve and $(E, C, g)$ a nontrivial relatively minimal elliptic fibration with section. We use the notation $B_{S} / K_{S}$ introduced in Definition 3.6. Then the following two conditions are equivalent:

(1) There exists a relatively minimal elliptic fibration $(X, C, f)$ satisfying the following conditions:

(a) The orbifold associated to $f$ is isomorphic to $(C, B)$ (Definition 4.23).

(b) The Jacobian fibration of $f$ is given by $g$.

(2) The following conditions are satisfied for any closed point $s$ on $C$ :

(a) If $g^{-1}(s)$ is not of type $\mathrm{I}_{n}(n \geq 0)$, then $B_{s}=K_{s}$.

(b) If $p>0$ and $g^{-1}(s)$ is isomorphic to a supersingular elliptic curve, then $p \nmid\left[B_{S}: K_{S}\right]$.

Proof. The theorem follows from Propositions 6.16 and 6.18 .

Proposition 6.20. Let $(X, C, f)$ be a relatively minimal elliptic fibration with Jacobian fibration $(E, C, g)$. Then the following conditions are equivalent:

(1) $\chi\left(\mathbb{O}_{X}\right) \leq 0$.

(2) $\chi\left(O_{X}\right)=0$.

(3) The reduction of any closed fiber of $f$ is isomorphic to an elliptic curve.

(4) $g$ is smooth.

Proof. The equivalence of (1) and (2) follows from Proposition 2 in [Mitsui 2014]. The equivalence of (2) and (3) follows from Proposition 2 in [Mitsui 2013]. The equivalence of (3) and (4) follows from Proposition 6.4.

Corollary 6.21. Let $(C, B)$ be a connected cyclic orbifold $k$-curve. Then there exists a relatively minimal elliptic fibration $(X, C, f)$ with $\chi\left(0_{X}\right)>0$ such that the orbifold associated to $f$ is isomorphic to $(C, B)$ (Definition 4.23).

Proof. Take a relatively minimal elliptic fibration $(E, C, g)$ satisfying the following conditions: (1) $g$ is not smooth; (2) $g$ admits a section; (3) for any $s \in \operatorname{Supp} B$, the closed fiber $g^{-1}(s)$ is isomorphic to an ordinary elliptic curve. Since the elliptic fibration $(E, C, g)$ is nontrivial, Theorem 6.19 gives a relatively minimal elliptic fibration $(X, C, f)$ such that the orbifold associated to $f$ is isomorphic to $(C, B)$. Proposition 6.20 shows that the inequality $\chi\left(0_{X}\right)>0$ holds. 
6D. Fundamental groups of elliptic surfaces. In this subsection, we use the following notation. Let $k$ be an algebraically closed field of characteristic $p \geq 0$, $C$ a connected proper smooth $k$-curve with function field $K$, and $(X, C, f)$ an elliptic fibration. We denote the intersection number of divisors $D_{1}$ and $D_{2}$ on $X$ by $D_{1} \cdot D_{2}$.

Lemma 6.22. Assume that two sections $D_{1}$ and $D_{2}$ of $f$ satisfy the following:

(1) $\left.\mathrm{O}_{X}\left(D_{1}-D_{2}\right)\right|_{X_{K}}$ is torsion in $\operatorname{Pic}\left(X_{K}\right)$, where $X_{K}$ is the generic fiber of $f$.

(2) For any closed point $x$ on $C$, there exists an irreducible component of the fiber $f^{-1}(x)$ that intersects with both of $D_{1}$ and $D_{2}$, and any other irreducible component of the fiber $f^{-1}(x)$ is disjoint from both of $D_{1}$ and $D_{2}$.

Then the equality $D_{1} \cdot D_{2}=-\chi\left(\mathscr{O}_{X}\right)$ holds.

Proof. First, we assume that $D_{1}=D_{2}$. Put $D:=D_{1}=D_{2}$ and $\mathscr{F}_{F}:=\mathscr{O}_{X}(D) / \mathscr{O}_{X}$. Since the genus of $X_{K}$ is equal to one and the effective divisor $\left.D\right|_{X_{K}}$ on $X_{K}$ is of degree one, the long exact sequence induced by the functor $f_{*}$ and the exact sequence of $\mathrm{O}_{X}$-modules

$$
0 \longrightarrow \mathrm{O}_{X} \longrightarrow \mathrm{O}_{X}(D) \longrightarrow \mathscr{F} \longrightarrow 0
$$

gives an isomorphism $f_{*} \mathscr{F}_{F} \rightarrow R^{1} f_{*} \mathrm{O}_{X}$. In particular, the equalities

$$
D \cdot D=\left.\operatorname{deg} \mathscr{O}_{X}(D)\right|_{D}=\operatorname{deg} f_{*} \mathscr{F}=\operatorname{deg} R^{1} f_{*} \mathscr{O}_{X}
$$

hold. The Riemann-Roch theorem for the line bundle $R^{1} f_{*} O_{X}$ on $C$ and the Leray spectral sequence for $f$ give the equalities

$$
\operatorname{deg} R^{1} f_{*} \mathscr{O}_{X}=\chi\left(R^{1} f_{*} \mathscr{O}_{X}\right)-\chi\left(\mathscr{O}_{C}\right)=-\chi\left(\mathscr{O}_{X}\right) .
$$

Thus, the equality $D \cdot D=-\chi\left(\mathrm{O}_{X}\right)$ holds.

Next, we consider the general case. By $n$ we denote the order of $\left.\mathcal{O}_{X}\left(D_{1}-D_{2}\right)\right|_{X_{K}}$ in $\operatorname{Pic}\left(X_{K}\right)$ (Condition (1)). Then $n\left(D_{1}-D_{2}\right)$ is linearly equivalent to a vertical divisor $V$. Condition (2) gives the equality $D_{0} \cdot\left(D_{1}-D_{2}\right)=0$ for any vertical prime divisor $D_{0}$. Thus, the equality $V \cdot V=0$ holds, which gives the equality $\left(D_{1}-D_{2}\right) \cdot\left(D_{1}-D_{2}\right)=0$. Therefore, the first case shows the general case.

Theorem 6.23. Choose a smooth closed fiber $i: X_{0} \rightarrow X$ of $f$. Take a geometric point $\bar{x}_{0}$ on $X_{0}$. Put $\bar{x}:=i\left(\bar{x}_{0}\right)$ and $\bar{s}:=f(\bar{x})$. By $(C, B)$ we denote the connected proper orbifold $k$-curve associated to $f$ (Definition 4.23). The morphisms $i$ and $f$ induce canonical homomorphisms $i_{*}: \pi_{1}\left(X_{0}, \bar{x}_{0}\right) \rightarrow \pi_{1}(X, \bar{x})$ and $f_{*}^{\text {orb }}: \pi_{1}(X, \bar{x}) \rightarrow \pi_{1}(C, B, \bar{s})$, respectively (Theorem 1.1). Then:

(1) If $\chi\left(O_{X}\right)>0$, then $i_{*}$ is trivial and $f_{*}^{\text {orb }}$ is an isomorphism.

(2) If $\chi\left(\mathrm{O}_{X}\right)=0$, then $i_{*}$ is injective. 
Remark 6.24. In the complex analytic case, Statement (2) does not hold in general for topological fundamental groups although Statement (1) holds and $i_{*}$ is nontrivial whenever $\chi\left(\mathscr{O}_{X}\right)=0$ [Friedman and Morgan 1994, 2.2.1 and 2.7.2]. For example, if $X$ is a Hopf surface, then $\chi\left(O_{X}\right)=0, \operatorname{Ker} i_{*} \cong \mathbb{Z}$, Coker $i_{*}=0$, and $\pi_{1}(X) \cong$ $\operatorname{Im} i_{*} \cong \mathbb{Z} \oplus \mathbb{Z} / n \mathbb{Z}$ for some positive integer $n$.

Proof. By $(E, C, g)$ we denote the Jacobian fibration of $f$. First, let us show Statement (1). We have only to show the following: for any connected étale covering space $\xi: Y \rightarrow X$, any connected component of $\xi^{-1}\left(X_{0}\right)$ is $X_{0}$-isomorphic to $X_{0}$. Assume that the above statement does not hold. Choose $\xi$ that does not satisfy the above statement. Take the Stein factorization

$$
Y \stackrel{h}{\longrightarrow} C^{\prime} \stackrel{u}{\longrightarrow} C
$$

of $f \circ \xi: Y \rightarrow X \rightarrow C$. Take the elliptic fibration $\left(X^{\prime}, C^{\prime}, f^{\prime}\right)$ and the étale morphisms $\xi^{\prime}: Y \rightarrow X^{\prime}$ and $u^{\prime}: X^{\prime} \rightarrow X$ given by Lemma 6.3. By assumption, the morphism $\xi^{\prime}$ is not an isomorphism. Replacing $\xi$ by $\xi^{\prime}$, we may assume that $(Y, C, f \circ \xi)$ is an elliptic fibration.

Since $\xi$ is étale, any closed fiber is of type ${ }_{m} \mathrm{I}_{n}(n \geq 0)$. Since $\chi\left(\mathrm{O}_{X}\right)>0$, Proposition 6.20 shows that $f$ admits a closed fiber of type ${ }_{m} \mathrm{I}_{n}(n>0)$. In particular, the $j$-invariant of $g$ is nonconstant (Proposition 6.4). By $d$ we denote the degree of $\xi$. If $p>0$, then $p \nmid d$ since $f$ admits a closed fiber that is isomorphic to a supersingular elliptic curve (Corollary 6.7) and $\xi$ is étale. Choose a connected proper smooth $k$-curve $C^{\prime}$ and a finite morphism $u: C^{\prime} \rightarrow C$ satisfying the following condition: the morphism $u$ induces an extension of the function fields $K^{\prime} / K$; by $J_{K}$ we denote the Jacobian of the generic fiber of $Y / C$; then $Y\left(K^{\prime}\right) \neq \varnothing$ and $J_{K}\left(K^{\prime}\right)$ contains $d^{2} d$-torsion elements. Take a desingularization $X^{\prime}$ of $X \times_{C} C^{\prime}$ and the canonical projection $u^{\prime}: X^{\prime} \rightarrow X$. Since $\xi^{\prime}$ is étale, the base change $\xi^{\prime}: Y^{\prime} \rightarrow X^{\prime}$ of $\xi$ via $u^{\prime}$ is étale. Thus, we obtain an étale $C^{\prime}$-morphism $\xi^{\prime \prime}: Y^{\prime \prime} \rightarrow X^{\prime \prime}$ between the minimal regular $C^{\prime}$-models of $Y^{\prime}$ and $X^{\prime}$ after successive blowing-downs of $(-1)$-curves on $Y^{\prime}$ and $X^{\prime}$. Replacing $\xi$ by $\xi^{\prime \prime}$, we may assume that $\xi$ is a morphism between Jacobian fibrations and $Y(K)$ contains $d^{2} d$-torsion elements.

By $H$ we denote the image of the $d$-torsion elements of $Y(K)$ under $\xi$. By $\bar{H}$ we denote the set of the closures of all elements of $H$ in $X$. Proposition 6.17 shows the following: (a) $\# H=d>1$; (b) all elements of $\bar{H}$ are disjoint; (c) for any closed point $x$ on $C$, there exists an irreducible component of the fiber $f^{-1}(x)$ that intersects with all elements of $\bar{H}$, and any other irreducible component of the fiber $f^{-1}(x)$ is disjoint from all elements of $\bar{H}$. This contradicts Lemma 6.22 since $\chi\left(O_{X}\right)>0$. Therefore the homomorphism $i_{*}$ is trivial.

Next, let us show Statement (2). We have only to show that, for any connected étale covering space $\tau: X_{0}^{\prime} \rightarrow X_{0}$, there exists an étale covering $\xi: Y \rightarrow X$ such that 
any connected component of the preimage $\xi^{-1}\left(X_{0}\right)$ is $X_{0}$-isomorphic to $X_{0}^{\prime}$. Since $\chi\left(O_{X}\right)=0$, Proposition 6.20 shows that $g$ is smooth. Take an integer $n \geq 3$ so that $p \nmid n$. Since the $n$-torsion $C$-subgroup scheme of $E / C$ is finite and étale, we may take a connected étale covering space $u: C^{\prime} \rightarrow C$ satisfying the following condition: let $u^{\prime}: E^{\prime} \rightarrow E$ denote the base change of $u$ via $g$; then $E^{\prime}\left(C^{\prime}\right)$ contains $n^{2} n$ torsion elements. Since $g$ is smooth, the $j$-invariant of $E$ is contained in $k$. Thus, the elliptic curve $E^{\prime}$ over $C^{\prime}$ induces a constant morphism from $C^{\prime}$ to the moduli scheme of elliptic curves with level $n$. Therefore, we obtain a $C^{\prime}$-isomorphism $E^{\prime} \cong X_{0} \times{ }_{k} C^{\prime}$. Take the base change $\tau^{\prime}: E^{\prime \prime} \rightarrow E^{\prime}$ of $\tau$ via the structure morphism $C^{\prime} \rightarrow$ Spec $k$. Take the $C$-morphism $h: X \rightarrow E$ given by Lemma 6.6. Then the base change of $u^{\prime} \circ \tau^{\prime}$ via $h$ is the desired morphism $\xi$.

Lemma 6.25. Let $R$ be a strictly Henselian excellent discrete valuation ring of equicharacteristic. By $\widehat{R}$ we denote the completion of $R$ with respect to the maximal ideal. Put $K:=\operatorname{Frac} R$ and $\widehat{K}:=$ Frac $\widehat{R}$. Let $\widehat{L} / \widehat{K}$ be a finite Galois extension. Then there exists a unique extension $L / K$ in $\widehat{L}$ such that $\widehat{L}=\widehat{K} L$. Furthermore, the extension $L / K$ is Galois, of degree $[\widehat{L}: \widehat{K}]$, and linearly disjoint from $\widehat{K} / K$.

Proof. Since $R$ is algebraically closed in $\widehat{R}$ by the approximation property [Bosch et al. 1990, 3.6.9], we have only to show the existence of $L$. We denote the characteristic of $R$ by $l$. Put $d:=[\widehat{L}: \hat{K}]$. By assumption, the extension $\widehat{L} / \widehat{K}$ is solvable. Thus, by induction on $d$, we may assume that $l \nmid d$ or $d=l>0$. The case $l \nmid d$ follows from Kummer theory since $R$ contains a primitive $d$-th root of unity. The case $d=l>0$ follows from Lemma 5.9(2) and Artin-Schreier theory.

Finally, we give a proof of the criterion for simple-connectedness of elliptic surfaces:

Proof of Theorem 1.2. We use the notation introduced in Theorem 6.23. Theorems 1.1 and 6.23 show that $\pi_{1}(X)$ is trivial if and only if $\pi_{1}(C, B)$ is trivial and $\chi\left(\mathscr{O}_{X}\right)>0$. Proposition 6.11 shows that the orbifold $(C, B)$ is cyclic. Thus, Theorem 1.3 shows that $\pi_{1}(C, B)$ is trivial if and only if $C \cong \mathbb{P}_{k}^{1}, B^{t}=B$, \# Supp $B \leq 2$, and $\operatorname{gcd}\left(n_{s}, n_{t}\right)=1$ for $s \neq t$, where we put $n_{s}:=\left[B_{s}: K_{s}\right]$ for each $s \in \operatorname{Supp} B$. Lemma 6.25 and Propositions 4.6, 6.11, and 6.15 imply that the above conditions on $(C, B)$ are equivalent to Conditions (2)-(6).

Let us show that each of Conditions (1)-(6) is necessary. We remark that $\chi\left(O_{X}\right)>0$ if and only if the Jacobian fibration of $f$ is not smooth (Proposition 6.20). The necessity of Conditions (1) and (2) is clear. The necessity of Conditions (3) and (4) follows from Corollary 6.21. The necessity of Conditions (5) and (6) follows from Proposition 6.15 and Theorem 6.19. 


\section{Appendix A: Triangle groups and projective special linear groups}

The result of this section is used in the proof of Proposition 5.4. Let $a, b$, and $c$ be integers greater than 1 . We define the triangle group $\Delta(a, b, c)$ by

$$
\Delta(a, b, c):=\left\langle x, y, z \mid x^{a}=y^{b}=z^{c}=x y z=\mathrm{id}\right\rangle .
$$

Let $p$ be a prime number and $q$ a power of $p$. In this section, we study homomorphisms $\Delta(a, b, c) \rightarrow \operatorname{SL}\left(2, \mathbb{F}_{q}\right)$ and $\Delta(a, b, c) \rightarrow \operatorname{PSL}\left(2, \mathbb{F}_{q}\right)$ that preserve the orders of $x, y$, and $z$. Take an algebraic closure $\overline{\mathbb{F}}_{q}$ of $\mathbb{F}_{q}$. For each positive integer $n$ prime to $p$, we take a primitive $n$-th root of unity $\zeta_{n}$ in $\overline{\mathbb{F}_{q}}$. Put $\mu_{n}:=\zeta_{n}+\zeta_{n}^{-1}$. The proofs of Lemmas A.1-A.4 are straightforward.

Lemma A.1. $\mu_{n} \in \mathbb{F}_{q}$ if and only if $n \mid\left(q^{2}-1\right)$.

Lemma A.2. We have equalities \# $\operatorname{SL}\left(2, \mathbb{F}_{q}\right)=q\left(q^{2}-1\right)$ and $\# \operatorname{PSL}\left(2, \mathbb{F}_{q}\right)=$ $q\left(q^{2}-1\right) / \operatorname{gcd}(2, p-1)$. The projection $\operatorname{SL}\left(2, \mathbb{F}_{q}\right) \rightarrow \operatorname{PSL}\left(2, \mathbb{F}_{q}\right)$ maps any element of order $n$ to an element of order $n / \operatorname{gcd}(2, p-1, n)$.

Lemma A.3. Take $X \in \operatorname{SL}\left(2, \mathbb{F}_{q}\right)$. Then the image of $X$ in $\operatorname{SL}\left(2, \overline{\mathbb{F}}_{q}\right)$ is conjugate to

$$
\left(\begin{array}{ll}
1 & 1 \\
0 & 1
\end{array}\right), \quad\left(\begin{array}{cc}
-1 & 1 \\
0 & -1
\end{array}\right), \quad \text { or } \quad\left(\begin{array}{cc}
\alpha & 0 \\
0 & \alpha^{-1}
\end{array}\right),
$$

where $\alpha \in \overline{\mathbb{F}}_{q} \times$. By $n$ we denote the order of $\alpha$ in $\overline{\mathbb{F}}_{q} \times$. If $p \neq 2$, then the order of $X$ is equal to $p, 2 p$, or $n$, respectively. Otherwise, the order of $X$ is equal to 2, 2, or $n$, respectively. The order of the image of $X$ in $\operatorname{PSL}\left(2, \mathbb{F}_{q}\right)$ is equal to $p, p$, or $n / \operatorname{gcd}(2, p-1, n)$, respectively.

Lemma A.4. Take $X \in \mathrm{SL}\left(2, \mathbb{F}_{q}\right)$. Let $n$ be an integer prime to $p$. Assume that $n>2$ and $\operatorname{tr} X=\mu_{n}$. Then the order of $X$ is equal to $n$.

Lemma A.5. Assume that $a, b$, and $c$ are greater than 2 and divide $q^{2}-1$. Then there exist $X, Y$, and $Z$ in $\operatorname{SL}\left(2, \mathbb{F}_{q}\right)$, of orders $a, b$, and $c$, respectively, such that $X Y Z=I$, where $I$ is the identity matrix of $\operatorname{SL}\left(2, \mathbb{F}_{q}\right)$.

Proof. Lemma A.1 shows that $\mu_{a}, \mu_{b}$, and $\mu_{c}$ are contained in $\mathbb{F}_{q}$. Put

$$
X:=\left(\begin{array}{cc}
0 & -1 \\
1 & \mu_{a}
\end{array}\right) \in \operatorname{SL}\left(2, \mathbb{F}_{q}\right)
$$

We have only to construct $Y$ and $Z$ in $\operatorname{SL}\left(2, \mathbb{F}_{q}\right)$ so that $\operatorname{tr} Y=\mu_{b}, \operatorname{tr} Z=\mu_{c}$, and $X Y Z=1$ (Lemma A.4). We write

$$
Y=\left(\begin{array}{ll}
\alpha & \beta \\
\gamma & \delta
\end{array}\right)
$$


Then we have only to choose $\alpha, \beta, \gamma$, and $\delta$ in $\mathbb{F}_{q}$ so that $\alpha \delta-\beta \gamma=1, \alpha+\delta=\mu_{b}$, and $\beta-\gamma+\mu_{a} \delta=\mu_{c}$. Thus, we have only to show that there exists a solution $(\alpha, \beta) \in \mathbb{F}_{q} \times \mathbb{F}_{q}$ of the equation $F(\alpha, \beta)=0$, where we put

$$
F(u, v):=u^{2}-\mu_{a} u v+v^{2}-\mu_{b} u+\left(\mu_{a} \mu_{b}-\mu_{c}\right) v+1 .
$$

If elements $\alpha_{0}, \alpha_{1}, \beta_{0}$, and $\beta_{1}$ in $\mathbb{F}_{q}$ satisfy $\alpha=\alpha_{0}+\alpha_{1}$ and $\beta=\beta_{0}+\beta_{1}$, then the equality

$$
F(\alpha, \beta)=F\left(\alpha_{0}, \beta_{0}\right)+\frac{\partial F}{\partial u}\left(\alpha_{0}, \beta_{0}\right) \alpha_{1}+\frac{\partial F}{\partial v}\left(\alpha_{0}, \beta_{0}\right) \beta_{1}+\alpha_{1}^{2}-\mu_{a} \alpha_{1} \beta_{1}+\beta_{1}^{2}
$$

holds. Note that the equalities

$$
\left\{\begin{array}{l}
(\partial F / \partial u)(u, v)=2 u-\mu_{a} v-\mu_{b} \\
(\partial F / \partial v)(u, v)=-\mu_{a} u+2 v+\mu_{a} \mu_{b}-\mu_{c}
\end{array}\right.
$$

hold. Since $a>2$ and $\operatorname{gcd}(p, a)=1$, the inequality $\mu_{a}^{2} \neq 4$ holds. Thus, we may take $\left(\alpha_{0}, \beta_{0}\right) \in \mathbb{F}_{q} \times \mathbb{F}_{q}$ so that $(\partial F / \partial u)\left(\alpha_{0}, \beta_{0}\right)=(\partial F / \partial v)\left(\alpha_{0}, \beta_{0}\right)=0$. Therefore, we have only to show that there exists a solution $\left(\alpha_{1}, \beta_{1}\right) \in \mathbb{F}_{q} \times \mathbb{F}_{q}$ of the equation $G\left(\alpha_{1}, \beta_{1}\right)=-F\left(\alpha_{0}, \beta_{0}\right)$, where we put $G(u, v):=u^{2}-\mu_{a} u v+v^{2}$. If $p \neq 2$ (resp. $p=2$ ), then the quadratic form $G(u, v)$ is nondegenerate (resp. nondefective), which concludes the proof.

Lemma A.6. Assume that the following conditions are satisfied:

(1) If $p \neq 2$, then $a=p$ or $2 p$.

(2) If $p=2$, then $a=2$.

(3) $b$ and $c$ are greater than 2 and divide $q^{2}-1$.

Then there exist $X, Y$, and $Z$ in $\operatorname{SL}\left(2, \mathbb{F}_{q}\right)$ of orders $a, b$, and $c$, respectively, such that $X Y Z=I$, where $I$ is the identity matrix of $\operatorname{SL}\left(2, \mathbb{F}_{q}\right)$.

Proof. Lemma A.1 shows that $\mu_{b}$ and $\mu_{c}$ are contained in $\mathbb{F}_{q}$. First, we consider the case $a=p$. We define $X, Y$, and $Z$ in $\operatorname{SL}\left(2, \mathbb{F}_{q}\right)$ by

$$
X:=\left(\begin{array}{ll}
1 & 1 \\
0 & 1
\end{array}\right), \quad Y:=\left(\begin{array}{cc}
\zeta_{b} & 0 \\
\mu_{c}-\mu_{b} & \zeta_{b}^{-1}
\end{array}\right), \quad \text { and } \quad Z:=\left(\begin{array}{cc}
\zeta_{b}^{-1} & -\zeta_{b}^{-1} \\
\mu_{b}-\mu_{c} & \mu_{c}-\zeta_{b}^{-1}
\end{array}\right) .
$$

Then the order of $X$ is equal to $p$. Moreover, the equalities $\operatorname{tr} Y=\mu_{b}, \operatorname{tr} Z=\mu_{c}$, and $X Y Z=I$ hold. Thus, the elements $X, Y$, and $Z$ satisfy the desired conditions (Lemma A.4). Next, we consider the case $p \neq 2$ and $a=2 p$. In that case, we have only to replace $X, Y$, and $Z$ by

$$
X:=\left(\begin{array}{cc}
-1 & 1 \\
0 & -1
\end{array}\right), \quad Y:=\left(\begin{array}{cc}
\zeta_{b} & 0 \\
\mu_{b}+\mu_{c} & \zeta_{b}^{-1}
\end{array}\right), \text { and } Z:=\left(\begin{array}{cc}
-\zeta_{b}^{-1} & -\zeta_{b}^{-1} \\
\mu_{b}+\mu_{c} & \zeta_{b}^{-1}+\mu_{c}
\end{array}\right)
$$


Proposition A.7. Assume that $a, b$, and $c$ are pairwise coprime. Take the elements $x, y$, and $z$ of $\Delta(a, b, c)$ in the definition of $\Delta(a, b, c)$. Put $G:=\operatorname{SL}\left(2, \mathbb{F}_{q}\right)$ or $\operatorname{PSL}\left(2, \mathbb{F}_{q}\right)$. Then there exists a homomorphism $\phi: \Delta(a, b, c) \rightarrow G$ such that the orders of $\phi(x), \phi(y)$, and $\phi(z)$ are equal to $a, b$, and $c$, respectively, if and only if the following three conditions are satisfied:

(1) $a b c \mid \# G$.

(2) If $G=\operatorname{SL}\left(2, \mathbb{F}_{q}\right)$ and one of $a, b$, and $c$ is equal to 2 , then $p=2$.

(3) If an integer $u$ is equal to $a, b$, or $c$ and is divisible by $p$, then $u$ satisfies one of the following conditions: (a) $u=p$ : (b) $G=\operatorname{SL}\left(2, \mathbb{F}_{q}\right), p \neq 2$, and $u=2 p$.

Proof. First, let us show the "only if" part. Since $a, b$, and $c$ are pairwise coprime, the condition on $\phi$ implies Condition (1). Lemma A.3 and the condition on $\phi$ imply Condition (3). Assume that $G=\operatorname{SL}\left(2, \mathbb{F}_{q}\right), a=2$, and $p \neq 2$. Then $\phi(x)=-I$, where $I$ is the identity matrix of $\operatorname{SL}\left(2, \mathbb{F}_{q}\right)$. Thus, the equality $\phi(y)=-\phi(z)$ holds, which contradicts the assumption that $b$ is prime to $c$. Therefore, Condition (2) holds. The "if" part follows from Lemmas A.2, A.5, and A.6.

Lemma A.8. Assume that $a, b$, and $c$ are pairwise coprime. Let $G$ be a nontrivial finite group. If there exists a surjective homomorphism $\Delta(a, b, c) \rightarrow G$, then $G$ is nonsolvable.

Proof. Assume that $G$ is solvable. Then there exists a nontrivial cyclic group $H$ and a surjective homomorphism $\phi: \Delta(a, b, c) \rightarrow H$. We may write $H=\mathbb{Z} / n \mathbb{Z}$, where $n$ is an integer greater than 1 . Take the elements $x, y$, and $z$ of $\Delta(a, b, c)$ in the definition of $\Delta(a, b, c)$. The orders of $x, y$, and $z$ are equal to $a, b$, and $c$, respectively. By $\bar{a}, \bar{b}$, and $\bar{c}$ we denote the orders of $\phi(x), \phi(y)$, and $\phi(z)$, respectively. Then $\bar{a}|a, \bar{b}| b, \bar{c} \mid c$ and $\overline{a b c} \neq 1$. In particular, the integers $\bar{a}, \bar{b}$, and $\bar{c}$ are pairwise coprime. Since $x y z=\mathrm{id}$, the equality $\phi(x)+\phi(y)+\phi(z)=0$ holds, which contradicts the facts that $\bar{a}, \bar{b}$, and $\bar{c}$ are pairwise coprime and $\overline{a b c} \neq 1$. Therefore, the group $G$ is nonsolvable.

Theorem A.9. Assume that $a, b$, and c are pairwise coprime. If $\{a, b, c\}=\{2,3,5\}$, then we suppose that $p=5$. Otherwise, we suppose that $2 a b c \mid\left(p^{2}-1\right)$. Take the elements $x, y$, and $z$ of $\Delta(a, b, c)$ in the definition of $\Delta(a, b, c)$. Then there exists a surjective homomorphism $\phi: \Delta(a, b, c) \rightarrow \operatorname{PSL}\left(2, \mathbb{F}_{p}\right)$ such that the orders of $\phi(x)$, $\phi(y)$, and $\phi(z)$ are equal to $a, b$, and $c$, respectively. Furthermore, there exists a prime number $p$ such that $2 a b c \mid\left(p^{2}-1\right)$.

Remark A.10. If $q>3$, then the group $\operatorname{PSL}\left(2, \mathbb{F}_{q}\right)$ is noncommutative and simple.

Proof. Let us show the first statement. By Lemma A.2, we may take $\phi$ in Proposition A.7. We have only to show that $\phi$ is surjective. Lemma A.8 shows 
that the image of $\phi$ is nonsolvable. Thus, the case $\{a, b, c\}=\{2,3,5\}$ follows from the fact that any proper subgroup of $\operatorname{PSL}\left(2, \mathbb{F}_{5}\right)\left(\cong A_{5}\right)$ is solvable. Assume that $\{a, b, c\} \neq\{2,3,5\}$. By the classification of the subgroups of PSL(2, $\left.\mathbb{F}_{p}\right)$ [Dickson 1958 , p. 285, XII, 260], any nonsolvable subgroup of $\operatorname{PSL}\left(2, \mathbb{F}_{p}\right)$ is isomorphic to $\operatorname{PSL}\left(2, \mathbb{F}_{5}\right)$ or $\operatorname{PSL}\left(2, \mathbb{F}_{p}\right)$. Suppose that $\phi$ is not surjective. Then the image of $\phi$ is isomorphic to $\operatorname{PSL}\left(2, \mathbb{F}_{5}\right)$. Since the order of any nontrivial element in $\operatorname{PSL}\left(2, \mathbb{F}_{5}\right)\left(\cong A_{5}\right)$ is equal to 2,3 or 5 , the equality $\{a, b, c\}=\{2,3,5\}$ holds, which contradicts the assumption. Thus, the homomorphism $\phi$ is surjective. The last statement follows from Dirichlet's theorem on arithmetic progressions.

\section{Appendix B: Comparison between orbifolds and stacks}

Let $k$ be an algebraically closed field of characteristic $p \geq 0$. For a nonnegative integer $n$, a DM stack $\mathscr{Y}$ is said to be of dimension $n$ if an atlas of $\mathscr{Y}$ is of dimension $n$. A stack orbifold $\mathscr{S}$ is a locally Noetherian normal DM stack that admits an open dense substack that is isomorphic to a scheme. A stack orbifold $k$-curve $\mathscr{Y}$ is a separated smooth $k$-stack of dimension one that is a stack orbifold. In the following, we see that the notion of an orbifold $k$-curve coincides with the notion of a stack orbifold $k$-curve.

We construct a stack orbifold $k$-curve from an orbifold $k$-curve. Let $(S, B)$ be a connected orbifold $k$-curve. Put $S_{0}:=S \backslash$ Supp $B$. Take $s \in S$. Choose an affine open subset $U$ containing $s$. By $\left(U,\left.B\right|_{U}\right)$ we denote the orbifold obtained by restricting $(S, B)$ to $U$. We may take a Galois orbifold trivialization $U^{\prime} \rightarrow\left(U,\left.B\right|_{U}\right)$ of $\left(U,\left.B\right|_{U}\right)$ (Theorem 1.3). We may regard the open subscheme $U \cap S_{0}$ of $U$ as an open substack of the quotient stack $\left[U^{\prime} / G\right]$. Pasting $\left[U^{\prime} / G\right]$ for all $s \in S$, we obtain a stack orbifold $k$-curve with coarse moduli space $S$.

We construct an orbifold $k$-curve from a stack orbifold $k$-curve. Let $\mathscr{S}$ be a connected stack orbifold $k$-curve. Take an open dense substack $\mathscr{S}_{0}$ of $\mathscr{Y}$ that is isomorphic to a scheme $S_{0}$. Take the coarse moduli space $\lambda: \mathscr{S} \rightarrow S$ of $\mathscr{Y}$ [Rydh 2013]. We may regard $S_{0}$ as an open subscheme of $S$. Thus, the equality $\operatorname{deg} \lambda=1$ holds [Vistoli 1989, 1.15]. Therefore, the scheme $S$ is of finite type over $k$. Since $\mathscr{S}$ is connected and normal, the scheme $S$ is connected and normal. Since $k$ is perfect, the scheme $S$ is a connected smooth $k$-curve.

By $P(S)$ we denote the set of all closed points on $S$. Take $s \in P(S)$. By $\operatorname{Aut}(s)$ we denote the automorphism group of $s$. If $s \in S_{0}$, then $\operatorname{Aut}(s)$ is trivial. By $S_{s}$ and $\mathscr{Y}_{S}$ we denote the schemes obtained by the strict Henselizations of $S$ and $\mathscr{Y}$ at $s$, respectively [Laumon and Moret-Bailly 2000, 6.2.1]. Take the quotient stack $\phi_{s}: \mathscr{Y}_{s} \rightarrow T_{s}:=\left[\mathscr{Y}_{s} / \operatorname{Aut}(s)\right]$ and the canonical morphism $\mu_{s}: T_{s} \rightarrow \mathscr{Y}$ [Laumon and Moret-Bailly 2000, 6.2.1]. By $B_{s}, L_{s}$, and $K_{s}$, we denote the fields of rational functions of $\mathscr{Y}_{s}, T_{s}$, and $S_{S}$, respectively [Vistoli 1989, 1.14]. Since the composite 
$\lambda \circ \mu_{s}: T_{s} \rightarrow S$ induces an isomorphism $K_{S} \rightarrow L_{S}$, the composite $\lambda \circ \mu_{s} \circ \phi_{s}: \mathscr{Y}_{S} \rightarrow S$ induces a finite Galois extension $B_{S} / K_{S}$ with Galois group $\operatorname{Aut}(s)$.

Take a separable closure $\overline{K_{s}}$ of $K_{s}$. We embed $B_{s}$ in $\overline{K_{s}}$ over $K_{s}$. Since $B_{s} / K_{s}$ is Galois, the image does not depend on the choice of the embedding. We define a map $B$ on $P(S)$ by $s \mapsto B_{S} / K_{S}$ (Definition 3.6). Then $\operatorname{Supp} B$ is locally finite since $\mathscr{Y}_{0}$ is open dense in $\mathscr{Y}$. Thus, the pair $(S, B)$ is a connected orbifold $k$-curve.

Theorem B.1. The above two correspondences give an equivalence between the category of orbifold $k$-curves and orbifold (étale) $k$-morphisms and the category of stack orbifold $k$-curves and (étale) $k$-morphisms that induce qsc morphisms between coarse moduli spaces. In particular, the fundamental group of any orbifold $k$-curve coincides with the fundamental group of the corresponding stack orbifold $k$-curve in [Noohi 2004, §4].

Remark B.2. The theorem does not hold for general orbifolds. There exists an orbifold étale covering space of a trivial orbifold $(S, B)$ that is not an étale covering space of the scheme $S$ (Examples 3.24 and 4.16).

Theorem B.1 follows from the local structure theorem on DM stacks [Laumon and Moret-Bailly 2000, 6.2]. The detail of the proof of the theorem is left to the readers.

\section{Acknowledgments}

The author thanks the referee for helpful comments. He is grateful to Professor Yuichiro Hoshi, who suggested that we use normalization instead of Stein factorization in studies on homotopy exact sequences induced by nonproper morphisms. This work was supported by the Grant-in-Aid for JSPS (the Japan Society for the Promotion of Science) Fellows (24-1432), the Grant-in-Aid for Young Scientists (B) (25800018) from the JSPS, and the Grant-in-Aid for Scientific Research (S) (24224001) from the JSPS.

\section{References}

[Bosch et al. 1990] S. Bosch, W. Lütkebohmert, and M. Raynaud, Néron models, Ergebnisse der Mathematik und ihrer Grenzgebiete (3) 21, Springer, Berlin, 1990. MR 91i:14034 Zbl 0705.14001

[Chau 1983] T. C. Chau, "A note concerning Fox's paper on Fenchel's conjecture”, Proc. Amer. Math. Soc. 88:4 (1983), 584-586. MR 84m:20038 Zbl 0497.20035

[Cossec and Dolgachev 1989] F. R. Cossec and I. V. Dolgachev, Enriques surfaces, I, Progress in Mathematics 76, Birkhäuser, Boston, 1989. MR 90h:14052 Zbl 0665.14017

[Dickson 1958] L. E. Dickson, Linear groups: With an exposition of the Galois field theory, Dover, New York, 1958. MR 21 \#3488 Zbl 0082.24901

[EGA III 1 1961] A. Grothendieck, "Eléments de géométrie algébrique, III: Étude cohomologique des faisceaux cohérents, I", Inst. Hautes Études Sci. Publ. Math. 11 (1961), 5-167. MR 0163910 Zbl 0118.36206 
[EGA III 2 1963] A. Grothendieck, "Eléments de géométrie algébrique, III: Étude cohomologique des faisceaux cohérents, II”, Inst. Hautes Études Sci. Publ. Math. 17 (1963), 5-91. MR 0163911 Zbl 0122.16102

[EGA IV 2 1965] A. Grothendieck, "Eléments de géométrie algébrique, IV: Étude locale des schémas et des morphismes de schémas, II", Inst. Hautes Études Sci. Publ. Math. 24 (1965), 5-231. MR 0199181 Zbl 0135.39701

[EGA IV 3 1966] A. Grothendieck, "Eléments de géométrie algébrique, IV: Étude locale des schémas et des morphismes de schémas, III", Inst. Hautes Études Sci. Publ. Math. 28 (1966), 5-255. MR 0217086 Zbl 0144.19904

[Fox 1952] R. H. Fox, “On Fenchel's conjecture about F-groups”, Mat. Tidsskr. B. 1952 (1952), 61-65. MR 14,843c Zbl 0049.15404

[Friedman and Morgan 1994] R. Friedman and J. W. Morgan, Smooth four-manifolds and complex surfaces, Ergebnisse der Mathematik und ihrer Grenzgebiete (3) 27, Springer, Berlin, 1994. MR 95m:57046 Zbl 0817.14017

[Iitaka 1971] S. Iitaka, "Deformations of compact complex surfaces, III", J. Math. Soc. Japan 23 (1971), 692-705. MR 44 \#7598 Zbl 0219.32012

[Katsura and Ueno 1985] T. Katsura and K. Ueno, "On elliptic surfaces in characteristic p", Math. Ann. 272:3 (1985), 291-330. MR 87g:14040 Zbl 0553.14019

[Katsura and Ueno 1986] T. Katsura and K. Ueno, "Multiple singular fibres of type $\mathbf{G}_{\boldsymbol{a}}$ of elliptic surfaces in characteristic p", pp. 405-429 in Algebraic and topological theories (Kinosaki, 1984), edited by M. Nagata et al., Kinokuniya, Tokyo, 1986. MR 1102270 Zbl 0800.14019

[Kodaira 1963] K. Kodaira, “On compact analytic surfaces, II”, Ann. of Math. (2) 77 (1963), 563-626. MR 32 \#1730 Zbl 0118.15802

[Kodaira 1964] K. Kodaira, "On the structure of compact complex analytic surfaces, I", Amer. J. Math. 86 (1964), 751-798. MR 32 \#4708 Zbl 0137.17501

[Lang 1986] W. E. Lang, "An analogue of the logarithmic transform in characteristic p", pp. 337-340 in Proceedings of the 1984 Vancouver conference in algebraic geometry, edited by J. Carrell et al., CMS Conf. Proc. 6, Amer. Math. Soc., Providence, RI, 1986. MR 846026 Zbl 0601.14033

[Laumon and Moret-Bailly 2000] G. Laumon and L. Moret-Bailly, Champs algébriques, Ergebnisse der Mathematik und ihrer Grenzgebiete (3) 39, Springer, Berlin, 2000. MR 2001f:14006 Zbl 0945.14005

[Lieblich and Olsson 2010] M. Lieblich and M. Olsson, "Generators and relations for the étale fundamental group”, Pure Appl. Math. Q. 6:1 (2010), 209-243. MR 2011b:14055 Zbl 05679716

[Liu 2002] Q. Liu, Algebraic geometry and arithmetic curves, Oxford Graduate Texts in Mathematics 6, Oxford University Press, 2002. MR 2003g:14001 Zbl 0996.14005

[Liu et al. 2004] Q. Liu, D. Lorenzini, and M. Raynaud, "Néron models, Lie algebras, and reduction of curves of genus one”, Invent. Math. 157:3 (2004), 455-518. MR 2005m:14039 Zbl 1060.14037

[Matsumura 1989] H. Matsumura, Commutative ring theory, 2nd ed., Cambridge Studies in Advanced Mathematics 8, Cambridge University Press, 1989. MR 90i:13001 Zbl 0666.13002

[Mitsui 2013] K. Mitsui, "Logarithmic transformations of rigid analytic elliptic surfaces", Math. Ann. 355:3 (2013), 1123-1170. MR 3020156 Zbl 1285.14028

[Mitsui 2014] K. Mitsui, “On a question of Zariski on Zariski surfaces”, Math. Z. 276:1-2 (2014), 237-242. MR 3150201 Zbl 1284.14048

[Moishezon 1977] B. Moishezon, Complex surfaces and connected sums of complex projective planes, Lecture Notes in Mathematics 603, Springer, Berlin-New York, 1977. MR 58 \#10931 Zbl 0392.32015 
[Noohi 2004] B. Noohi, "Fundamental groups of algebraic stacks", J. Inst. Math. Jussieu 3:1 (2004), 69-103. MR 2004k:14003 Zbl 1052.14001

[Raynaud 1970] M. Raynaud, "Spécialisation du foncteur de Picard”, Inst. Hautes Études Sci. Publ. Math. 38 (1970), 27-76. MR 44 \#227 Zbl 0207.51602

[Raynaud 1990] M. Raynaud, " $p$-groupes et réduction semi-stable des courbes", pp. 179-197 in The Grothendieck Festschrift, III, edited by P. Cartier et al., Progress in Mathematics 88, Birkhäuser, Boston, 1990. MR 92m:14025 Zbl 0722.14013

[Raynaud 1994] M. Raynaud, "Revêtements de la droite affine en caractéristique $p>0$ et conjecture d'Abhyankar", Invent. Math. 116:1-3 (1994), 425-462. MR 94m:14034 Zbl 0798.14013

[Rydh 2013] D. Rydh, "Existence and properties of geometric quotients", J. Algebraic Geom. 22:4 (2013), 629-669. MR 3084720 Zbl 1278.14003

[Seifert 1933] H. Seifert, “Topologie Dreidimensionaler Gefaserter Räume”, Acta Math. 60:1 (1933), 147-238. MR 1555366 Zbl 0006.08304

[SGA 1 1971] A. Grothendieck, Revêtements étales et groupe fondamental (Séminaire de Géométrie Algébrique du Bois Marie 1960-1961), Lecture Notes in Math. 224, Springer, Berlin, 1971. MR 50 \#7129 Zbl 0234.14002

[SGA $4 \frac{1}{2}$ 1977] P. Deligne, Cohomologie étale (Séminaire de Géométrie Algébrique du Bois Marie), Lecture Notes in Math. 569, Springer, Berlin-New York, 1977. MR 57 \#3132 Zbl 0349.14008

[Silverman 2009] J. H. Silverman, The arithmetic of elliptic curves, 2nd ed., Graduate Texts in Mathematics 106, Springer, Dordrecht, 2009. MR 2010i:11005 Zbl 1194.11005

[Thornton 1967] M. C. Thornton, "Singularly fibered manifolds", Illinois J. Math. 11 (1967), 189-201. MR 35 \#1031 Zbl 0146.45104

[Thurston 1980] W. P. Thurston, "The Geometry and Topology of Three-Manifolds", lecture notes, 1980, http://library.msri.org/books/gt3m/.

[Ue 1986] M. Ue, "On the diffeomorphism types of elliptic surfaces with multiple fibers", Invent. Math. 84:3 (1986), 633-643. MR 87j:57019 Zbl 0595.14028

[Vistoli 1989] A. Vistoli, "Intersection theory on algebraic stacks and on their moduli spaces", Invent. Math. 97:3 (1989), 613-670. MR 90k:14004 Zbl 0694.14001

[Xiao 1991] G. Xiao, “ $\pi_{1}$ of elliptic and hyperelliptic surfaces", Internat. J. Math. 2:5 (1991), 599-615. MR 92j:14049 Zbl 0762.14013

Communicated by Shigefumi Mori

Received 2014-10-08 Revised 2015-01-21 Accepted 2015-03-11

mitsui@math.kobe-u.ac.jp Department of Mathematics, Graduate School of Science, Kobe University, Hyogo 657-8501, Japan 


\section{Algebra \& Number Theory}

msp.org/ant

\section{EDITORS}

MANAGING EDITOR

Bjorn Poonen

Massachusetts Institute of Technology

Cambridge, USA

\author{
EDITORIAL BOARD CHAIR \\ David Eisenbud \\ University of California \\ Berkeley, USA
}

BOARD OF EDITORS

Georgia Benkart

Dave Benson

Richard E. Borcherds

John H. Coates

J-L. Colliot-Thélène

Brian D. Conrad

Hélène Esnault

Hubert Flenner

Sergey Fomin

Edward Frenkel

Andrew Granville

Joseph Gubeladze

Roger Heath-Brown

Craig Huneke

Kiran S. Kedlaya

János Kollár

Yuri Manin

Philippe Michel
University of Wisconsin, Madison, USA

University of Aberdeen, Scotland

University of California, Berkeley, USA

University of Cambridge, UK

CNRS, Université Paris-Sud, France

Stanford University, USA

Freie Universität Berlin, Germany

Ruhr-Universität, Germany

University of Michigan, USA

University of California, Berkeley, USA

Université de Montréal, Canada

San Francisco State University, USA

Oxford University, UK

University of Virginia, USA

Univ. of California, San Diego, USA

Princeton University, USA

Northwestern University, USA

École Polytechnique Fédérale de Lausanne
Susan Montgomery

Shigefumi Mori

Raman Parimala

Jonathan Pila

Anand Pillay

Victor Reiner

Peter Sarnak

Joseph H. Silverman

Michael Singer

Vasudevan Srinivas

J. Toby Stafford

Ravi Vakil

Michel van den Bergh

Marie-France Vignéras

Kei-Ichi Watanabe

Efim Zelmanov

Shou-Wu Zhang
University of Southern California, USA

RIMS, Kyoto University, Japan

Emory University, USA

University of Oxford, UK

University of Notre Dame, USA

University of Minnesota, USA

Princeton University, USA

Brown University, USA

North Carolina State University, USA

Tata Inst. of Fund. Research, India

University of Michigan, USA

Stanford University, USA

Hasselt University, Belgium

Université Paris VII, France

Nihon University, Japan

University of California, San Diego, USA

Princeton University, USA

\section{PRODUCTION}

production@msp.org

Silvio Levy, Scientific Editor

See inside back cover or msp.org/ant for submission instructions.

The subscription price for 2015 is US $\$ 255 /$ year for the electronic version, and $\$ 440 /$ year ( $+\$ 55$, if shipping outside the US) for print and electronic. Subscriptions, requests for back issues and changes of subscribers address should be sent to MSP.

Algebra \& Number Theory (ISSN 1944-7833 electronic, 1937-0652 printed) at Mathematical Sciences Publishers, 798 Evans Hall \#3840, c/o University of California, Berkeley, CA 94720-3840 is published continuously online. Periodical rate postage paid at Berkeley, CA 94704, and additional mailing offices.

ANT peer review and production are managed by EditFLOW ${ }^{\circledR}$ from MSP.

\section{PUBLISHED BY}

- mathematical sciences publishers

nonprofit scientific publishing

http://msp.org/

(C) 2015 Mathematical Sciences Publishers 


\section{Algebra \& Number Theory}

\section{Volume $9 \quad$ No. $5 \quad 2015$}

$p$-adic Hodge-theoretic properties of étale cohomology with mod $p$ coefficients, and the 1035 cohomology of Shimura varieties

MATTHEW EMERTON and Toby GeE

Homotopy exact sequences and orbifolds

KENTARO Mitsui

Factorially closed subrings of commutative rings

SAGNIK CHAKRABORTY, RAJENDRA VASANT GURJAR and MASAYOSHI MIYANISHI

Coherent analogues of matrix factorizations and relative singularity categories 